\title{
Las asignaturas de creatividad $y$ estrategia en los Grados en Comunicación en España
}

\section{The subjects of creativity and strategy in degrees in Communication in Spain}

\author{
Araceli Castelló-Martínez. Universidad de Alicante. España. \\ araceli.castello@ua.es \\ $[\mathrm{CV}](\mathrm{C}$
}

La investigación que presenta este artículo se enmarca dentro del grupo de investigación "Comunicación y Públicos Específicos (COMPUBES)", financiado por la Universidad de Alicante y desarrollado bajo la dirección de la Profesora Doctora Victoria Tur-Viñes.

\author{
Cómo citar este artículo / Referencia normalizada \\ Castelló-Martínez, A. (2020). Las asignaturas de creatividad y estrategia en los Grados en \\ Comunicación en España. Revista Latina de Comunicación Social, (77), 143-178. \\ https://www.doi.org/10.4185/RLCS-2020-1453
}

\section{RESUMEN}

El modelo de enseñanza basado en la formación en competencias que instauró el Espacio Europeo de Educación Superior (EEES) está enfocado a adecuar la formación universitaria a las necesidades del mercado laboral. El presente artículo analiza las competencias creativa y estratégica entre las competencias y los perfiles propuestos por ANECA (2005) para los Grados en Comunicación en España. En una segunda fase, se realiza una revisión de las asignaturas relacionadas con la estrategia creativa dentro los planes de estudio de las titulaciones en Comunicación, a partir de una muestra de 125 titulaciones y 202 asignaturas. Los resultados demuestran la escasa presencia de la estrategia creativa en los planes de estudio en Comunicación, siendo los Grados en Publicidad y Relaciones Públicas las titulaciones en las que más aparece, así como la falta de consenso existente en las nomenclaturas de las asignaturas.

PALABRAS CLAVE: comunicación; publicidad y relaciones públicas; creatividad; estrategia; estrategia creativa.

\begin{abstract}
Training in competency-based learning model that the European Higher Education Area (EHEA) established is focused on university education to adapt to the needs of the occupational market. This article examines the creative and strategic skills between competencies and profiles proposed by ANECA (2005) for Degrees in Communication in Spain. In a second phase, a review of the subjects related to the creative strategy within curricula of degrees in Communication is made, from a sample of 125 degrees and 202 subjects. The results demonstrate the limited presence of the creative strategy in the curricula in Communication, being the Degrees in Advertising and Public Relations where it more frequently appears, as well as the lack of consensus in the appellation of the subjects.
\end{abstract}


KEYWORDS: communication; advertising and public relations; creativity; stratey; creative strategy.

\section{CONTENIDOS}

1. Introducción. 1.1. Las competencias en el marco del Espacio Europeo de Educación Superior. 1.2. Las competencias creativa y estratégica en los estudios de Comunicación. 1.3. La creatividad y la estrategia en los perfiles profesionales de los estudios de Comunicación. 1.4. La enseñanza de la creatividad y la estrategia publicitarias. 1.5. La investigación sobre la enseñanza de la creatividad publicitaria. 1.6. La asignatura Estrategia Creativa y Conceptualización en el Grado en Publicidad y Relaciones Públicas de la Universidad de Alicante. 2. Método. 2.1. Estrategias metodológicas. 2.2. Población y muestra. 2.3. Instrumentos de recogida de información y procedimiento. 3. Resultados. 4. Discusión, 5. Conclusiones. 6. Referencias bibliográficas. 7. Referencias relacionadas.

\section{Introducción}

\subsection{Las competencias en el marco del Espacio Europeo de Educación Superior}

El proceso de convergencia y armonización europea en los sistemas de educación superior que se inició con la implantación del Espacio Europeo de Educación Superior (EEES) supuso la introducción de grandes novedades en el planteamiento de la enseñanza universitaria, con el objetivo de alcanzar un territorio común que favoreciera el crecimiento y la cohesión social a partir de la formación de la ciudadanía. Pero, sobre todo, ha conllevado un continuo "repensar" la universidad (Marta-Lazo, 2009, p. 3), un replanteamiento de sus métodos docentes por parte del profesorado y una constante revisión del trabajo realizado por los alumnos fuera del aula para adecuar las asignaturas a un sistema más interactivo, enfocado al protagonismo del alumno en el proceso de aprendizaje y a su participación plenamente activa.

La filosofía del EEES redefine la tarea de la institución universitaria, en el marco de una sociedad globalizada, hipermediatizada y supertecnificada. La docencia de calidad que abandona el concepto de "educación bancaria" (Freire, 1995) para fundamentarse en el de "aprendizaje experiencial" (Kolb, 1984) con orientación profesionalizante, la integración de las herramientas digitales, las técnicas pedagógicas de aprendizaje activo centradas en el alumno y el fomento de la movilidad internacional son sus principales características.

Por otra parte, el enfoque del EEES está encaminado a la inserción laboral del alumno, reivindica el carácter eminentemente práctico de los estudios, reclama una metodología basada en aprender a aprender lo que sea necesario para el desempeño profesional, lo cual ha favorecido que la Educación Superior se acerque al ámbito empresarial y se inspira en el Proceso de Aprendizaje Permanente (PAP $)^{1}$, el aprender para seguir aprendiendo a lo largo de la vida: "Un planteamiento demandado por las nuevas exigencias de la Sociedad del Conocimiento, en la que se precisa una continua actualización de los aprendizajes que permita la adaptación a los rápidos cambios y transformaciones que van sucediendo" (Pérez, Vadillo y McMahon, 2011, pp. 68-69).

Los nuevos modelos de enseñanza en el seno del EEES se caracterizan por centrarse en el aprendizaje autónomo del alumno expresado en competencias para el perfil profesional

\footnotetext{
${ }^{1}$ El concepto de PAP -en inglés, Long Life Learning (LLL)-, fue impulsado por la UNESCO tras la presentación en 1996 del Informe Delors "Learning: The Treasure Wihtin", en el que se insta a que las reformas educativas y las nuevas políticas pedagógicas se orienten en la "educación a lo largo de la vida", centrada en cuatro pilares: "aprender a conocer, aprender a hacer, aprender a vivir justos y aprender a ser".
} 
correspondiente y tutorizado por profesores con un enfoque cooperativo del proceso de enseñanzaaprendizaje (Fernández, 2005).

En la sociedad actual el modelo de aprendizaje en el que se basa el sistema académico apuesta por la adquisición de competencias hacia la empleabilidad. Las capacidades y destrezas que fomentan las competencias y la apuesta por las TIC en el proceso de enseñanza-aprendizaje obligan a que el alumno "aprenda haciendo", por lo que los métodos de enseñanza tradicionales deben sustituirse por las llamadas metodologías activas, en las que el alumno ocupa un papel protagonista y activo en la adquisición del conocimiento.

El acercamiento de la formación universitaria a las demandas del mercado laboral es uno de los objetivos de los planes de grado: promover una relación más directa y eficaz entre la enseñanza universitaria y la actividad profesional. La formación basada en competencias, que busca capacitar al alumno para ejercer con éxito la actividad profesional para la que cualifica el grado, destaca, en este sentido, "el acercamiento entre la universidad y la realidad empresarial", apostando "por una formación de tipo más práctica y por la resolución de problemas simulando una situación real del mercado laboral" (Sierra y Cabezuelo, 2010, p. 32).

En 2003 se llevó a cabo el proyecto piloto Tuning (González y Wagenaar, 2003) para establecer una metodología que permitiera la armonización de las estructuras educativas europeas en disciplinas concretas -matemáticas, geología, estudios empresariales, historia y ciencias de la educación-, en el que participaron 70 universidades europeas. Este proyecto destaca la necesidad de formar en competencias y desarrollar en los alumnos capacidades y conocimientos más allá de lo puramente técnico, lo que llevó a la definición de 32 competencias, denominadas transversales o genéricas, que todo titulado universitario debe adquirir, en mayor o menor medida, dependiendo de sus estudios. Marta-Lazo (2012, p. 19) clasifica las competencias genéricas o transversales incluidas en este proyecto en tres: instrumentales, interpersonales y sistémicas. 
RLCS, Revista Latina de Comunicación Social, 77, 143-178

[Investigación] DOI: 10.4185/RLCS-2020-1453 | ISSN 1138-5820 | Año 2020

Tabla 1. Clasificación de competencias genéricas o transversales en el proyecto Tuning.

\begin{tabular}{|l|}
\hline \multicolumn{1}{|c|}{ Clasificación de competencias } \\
\hline \multicolumn{1}{|c|}{ Instrumentales } \\
\hline 1 Capacidad de análisis y síntesis \\
2 Capacidad de organización, planificación y gestión del tiempo \\
3 Conocimiento general básico \\
4 Profundización en el conocimiento básico de la profesión \\
5 Comunicación oral y escrita en el idioma propio \\
6 Conocimiento de una o más lenguas extranjeras \\
7 Habilidades básicas informáticas \\
8 Conocimientos de informática relativos al ámbito de estudio \\
9 Habilidades de gestión de la información \\
10 Resolución de problemas \\
11 Toma de decisiones \\
\hline \multicolumn{1}{|c|}{ Interpersonales } \\
\hline 1 Trabajo en equipo \\
2 Trabajo en un equipo de carácter interdisciplinar \\
3 Capacidad para trabajar en un contexto internacional \\
4 Capacidad para comunicarse con expertos de otros campos \\
5 Habilidades en las relaciones interpersonales \\
6 Razonamiento crítico \\
7 Compromiso ético \\
8 Capacidad de crítica y auto-crítica \\
9 Apreciación de la diversidad y la multiculturalidad \\
\hline \multicolumn{1}{|c|}{ Sistémicas } \\
\hline 1 Capacidad para aplicar el conocimiento en la práctica \\
2 Capacidad de aprendizaje (adquirir experiencia) \\
3 Aprendizaje autónomo \\
4 Adaptación a nuevas situaciones \\
5 Liderazgo \\
6 Conocimiento de otras culturas y costumbres \\
7 Iniciativa y espíritu emprendedor \\
8 Motivación por la calidad \\
9 Sensibilidad hacia temas medioambientales \\
11 Capacidades de investigación \\
12 Diseño y gestión de proyectos \\
\hline
\end{tabular}

Fuente: elaboración propia a partir de Marta-Lazo (2012, p. 19).

Las 32 competencias genéricas que se propusieron en el proyecto Tuning fueron acogidas por la ANECA para la realización de los Libros Blancos de las diferentes titulaciones. A estas competencias hay que sumar las específicas, particulares de cada uno de los grados.

\subsection{Las competencias creativa y estratégica en los estudios de Comunicación}

Si bien el pensamiento creativo y estratégico es transversal y extensible a todos los conocimientos del futuro profesional de la comunicación, entre las competencias listadas por ANECA (2005) para cada titulación en Comunicación algunas hacen alusión directa a la creatividad y la estrategia. En el caso de Comunicación Audiovisual, entre las competencias disciplinares se encuentran (ANECA, 2005, pp. 229-270):

- Conocimiento y aplicación de las técnicas y procesos de creación y difusión audiovisuales en sus diversas fases, tanto en el cine como en televisión, vídeo y multimedia desde el punto de vista de la puesta en escena desde la idea inicial hasta su acabado final.

- Conocimiento, identificación y aplicación de recursos, elementos, métodos y procedimientos de los procesos de construcción y análisis de los relatos audiovisuales tanto lineales como no lineales, incluyendo el diseño, establecimiento y desarrollo de estrategias, así como las aplicaciones de las políticas de comunicación persuasiva en los mercados audiovisuales. 
En las competencias profesionales, académicas y otras de Comunicación Audiovisual también se especifican algunas vinculadas con las materias que nos ocupan:

- Profesionales. Capacidad para diseñar y concebir la presentación estética y técnica de la puesta en escena a través de las fuentes lumínicas y acústicas naturales o artificiales atendiendo a las características creativas y expresivas que propone el director del proyecto audiovisual.

- Académicas. Capacidad para definir temas de investigación o creación personal innovadora que puedan contribuir al conocimiento o desarrollo de los lenguajes audiovisuales o su interpretación.

- Otras. Creatividad: capacidad para asumir riesgos expresivos y temáticos en el marco de las disponibilidades y plazos de la producción audiovisual, aplicando soluciones y puntos de vista personales en el desarrollo de los proyectos.

- Otras. Toma de decisiones: capacidad para acertar al elegir en situaciones de incertidumbre, asumiendo responsabilidades.

Entre las competencias disciplinares de los estudios de Periodismo definidas por ANECA (2005, pp. 192-225) encontramos dos relacionadas con estas materias:

- Conocimiento de las teorías sobre la publicidad, las relaciones públicas y la comunicación corporativa, a partir de la aplicación del pensamiento creador y mediante sus diversas manifestaciones y actividades.

- Conocimiento de los principales debates y acontecimientos mediáticos derivados la coyuntura actual y de cómo se gestan y difunden según las estrategias comunicativas y los intereses de todo tipo.

En las competencias profesionales de esta titulación también se indica la capacidad de experimentar e innovar mediante el conocimiento y uso de técnicas y métodos aplicados a los procesos de mejora de la calidad y de auto evaluación, así como habilidades para el aprendizaje autónomo, la adaptación a los cambios y la superación rutinaria mediante la creatividad.

Centrándonos en la titulación en Publicidad y Relaciones Públicas, la creatividad aparece en cuatro competencias, como muestra la tabla 2.

Tabla 2. Competencias del grado en Publicidad y Relaciones Públicas relacionadas con la creatividad.

\begin{tabular}{|c|l|}
\hline Disciplinar & $\begin{array}{l}\text { 9. Conocimiento de los métodos del pensamiento creador y su aplicación en } \\
\text { el proceso de comunicación publicitaria: escuelas, métodos y técnicas. }\end{array}$ \\
Profesional & $\begin{array}{l}\text { 8. Capacidad y habilidad para dar forma creativa al mensaje. Sus principales } \\
\text { funciones abarcan la realización de las piezas publicitarias básicas y la } \\
\text { supervisión de su producción definitivas. }\end{array}$ \\
Académicas & $\begin{array}{l}\text { 4. Capacidad para la creatividad y la innovación: capacidad para evolucionar } \\
\text { hacia lo desconocido, partiendo de un sólido conocimiento de lo actual. }\end{array}$ \\
específicas & $\begin{array}{l}\text { 3. Capacidad de perspicacia, de ingenio y creatividad que le permita hallar } \\
\text { soluciones eficaces a problemas inéditos. Preparación para asumir el riesgo, } \\
\text { desarrollando la capacidad desde pensar a decidir. }\end{array}$ \\
\hline
\end{tabular}

Fuente: elaboración propia a partir de ANECA (2005, pp. 273-296). 
Cabe hacer una apreciación al respecto: si bien en la competencia académica 4 no se especifica ningún ámbito en concreto, sí se hace así en el conocimiento disciplinar 9 y el conocimiento profesional 8, que inciden en que la aplicación del pensamiento creador debe ejercerse en "el proceso de comunicación publicitaria: escuelas, métodos y técnicas" y se vinculan al ejercicio publicitario "sus principales funciones abarcan la realización de las piezas publicitarias básicas y la supervisión de su producción definitiva"-. En este sentido, es necesario despatrimonializar la creatividad como una aptitud exclusiva de la fase de creación del mensaje en el proceso publicitario y destacarla como un valor indispensable en el diseño no sólo de estrategias publicitarias, sino también de estrategias de relaciones públicas, en particular, y de comunicación, en general.

Estas pautas han hecho que, actualmente, la creatividad todavía esté íntimamente relacionada con el ámbito publicitario en los planes de estudios, a pesar de que la materia Creatividad e innovación en comunicación, especialmente publicitaria y de las relaciones públicas, propuesta por ANECA sí haga mención a los dos ámbitos. Como destaca Estanyol (2012, p. 16), un gran número de las asignaturas sobre creatividad que se ofrecen en las titulaciones de Grado en Publicidad y Relaciones Públicas en su denominación indican que están centradas en la publicidad $(46,67 \%)$ y sólo una minoría $(1,66 \%)$ está específicamente enfocada a su aplicación en relaciones públicas.

Paralelamente, también encontramos conocimientos directamente relacionados con la estrategia entre las competencias de los estudios en Publicidad y Relaciones Públicas, como muestra la tabla 3. En este caso, exceptuando la competencia disciplinar 12 -en la que se alude a los mensajes publicitariosen el resto no se especifica el ámbito de actuación. No encontramos competencias académicas directamente relacionadas con el enfoque estratégico.

Tabla 3. Competencias del grado en Publicidad y Relaciones Públicas relacionadas con la estrategia.

\begin{tabular}{|c|l|}
\hline Disciplinar & $\begin{array}{l}\text { 6. Desarrolla los fundamentos conceptuales de la dirección estratégica aplicados } \\
\text { a la comunicación por objetivos para, desde un planteamiento metodológico, } \\
\text { asistir a los operadores que tienen que tomar decisiones en búsqueda de la } \\
\text { eficacia comunicativa. } \\
\text { 12. Estudio de las estrategias y procesos encaminados a la creación y realización } \\
\text { de mensajes publicitarios en los distintos soportes y medios de comunicación. }\end{array}$ \\
Profesional & $\begin{array}{l}\text { 22. Conocimiento del diseño y desarrollo de las estrategias y aplicaciones de las } \\
\text { políticas de comunicación persuasiva, estudiando la metodología necesaria para } \\
\text { analizar el estado corporativo, evaluarlo mediante auditoría y diseñar una } \\
\text { estrategia específica. } \\
\text { 3. Capacidad y habilidad para establecer el plan de comunicación: fijar los } \\
\text { objetivos de comunicación, definir el público objetivo, plantear las estrategias y } \\
\text { controlar el presupuesto de comunicación. }\end{array}$ \\
Académicas & $\begin{array}{l}\text { Otras } \\
\text { específicas } \\
\text { relacionar las causas y los efectos. Saber gestionar el tiempo, con habilidad para la } \\
\text { organización y temporalización de las tareas. }\end{array}$ \\
\hline
\end{tabular}

Fuente: elaboración propia a partir de ANECA (2005, pp. 273-296).

Junto con las competencias para cada titulación, el Libro Blanco de los títulos de Grado en Comunicación elaborado por ANECA (2005) incluye los contenidos formativos mínimos de las 
titulaciones en Comunicación Audiovisual, Periodismo y Publicidad y Relaciones Públicas organizados por materias, de los que se derivan habilidades y destrezas. A partir de esta sistematización de las competencias de los futuros profesionales de la comunicación, ANECA (2005) también propone para cada titulación una serie de perfiles profesionales básicos.

Si revisamos la presencia de la creatividad y la estrategia entre las materias básicas de los Grados en Comunicación, encontramos que en la titulación de Comunicación Audiovisual existe una materia relacionada, en la de Periodismo no aparece ninguna y en los estudios de Publicidad y Relaciones Públicas se indican dos materias. Con respecto a los perfiles profesionales, encontramos dos perfiles relacionados en la titulación de Publicidad y Relaciones Públicas.

En el caso de la titulación de Comunicación Audiovisual, entre las materias básicas se indica la siguiente: Técnicas y procesos creativos de producción, realización y postproducción. Los contenidos formativos mínimos de esta materia son:

- Dirección audiovisual: cine, televisión, vídeo.

- Aplicaciones creativas básicas sobre escenografía, iluminación, dirección de fotografía, dirección de actores.

- Procesos de creación, registro y difusión de la producción radiofónica, discográfica y otros productos sonoros.

- Diseño de productos multimedia y entornos informáticos interactivos.

- Edición y posproducción sonora, audiovisual y multimedia.

Esta materia tiene un total de horas de trabajo del alumno estimadas de 750-900, siendo la práctica (40\%) y el trabajo personal (40\%) las actividades con un mayor peso. Las destrezas, habilidades y competencias a adquirir a partir de estos conocimientos son:

- capacidad y habilidad para gestionar los procesos de dirección-realización de los diversos medios audiovisuales (2).

- Habilidad para el manejo de las técnicas y procedimientos de composición fotográfica, cinematográfica, televisiva e hipermedia (1).

Dentro del diseño del plan los estudios en Publicidad y Relaciones Públicas propuesto por ANECA (2005), encontramos una materia relacionada con la creatividad y otra con la estrategia: Creatividad e innovación en comunicación, especialmente publicitaria y de las relaciones públicas y Fundamentos y técnicas de la estrategia de la comunicación (especialmente publicitaria y de las relaciones, públicas), respectivamente. En el primer caso, los contenidos formativos mínimos de la materia definida por ANECA (2005, pp. 337-338) para los Grados en Publicidad y Relaciones Públicas, con 650-780 horas de trabajo estimadas y un peso del trabajo personal del $40 \%$, son los siguientes:

- Conocimiento teórico-práctico de los elementos, formas y procesos de los discursos publicitarios y de otras formas de comunicación, y de su evolución.

- Conocimiento de los métodos del pensamiento creador y su aplicación en el proceso de comunicación, especialmente publicitaria y de las relaciones públicas: escuelas, métodos y técnicas.

- Conocimiento de los procesos de elaboración y análisis de los mensajes publicitarios y sus códigos.

- Estudio de las estrategias y procesos encaminados a la creación, producción y realización de mensajes publicitarios en los distintos soportes y medios de comunicación.

- Conocimiento del sistema de trabajo de los departamentos creativos: redacción, dirección de arte y producción.

- Estudio de la información escrita, audiovisual y digital como marco de referencia. 
A partir de estos objetivos, ANECA (2005, pp. 337-338) identifica las siguientes destrezas, habilidades y competencias a adquirir (nivel y profundidad):

Tabla 4. Destrezas, habilidades y competencias a adquirir (nivel y profundidad).

\begin{tabular}{|l|}
\hline $\begin{array}{l}\text { Capacidad para la creatividad y la innovación: capacidad para evolucionar hacia lo desconocido, } \\
\text { partiendo de un sólido conocimiento del proyecto y la estrategia a seguir (3). }\end{array}$ \\
\hline $\begin{array}{l}\text { Desarrollo de la capacidad de perspicacia, de ingenio y la creatividad para hallar soluciones eficaces a } \\
\text { problemas inéditos (2). }\end{array}$ \\
\hline Capacidad y habilidad para dar forma creativa al mensaje (2). \\
\hline $\begin{array}{l}\text { Capacidad y habilidad para la creación, diseño y desarrollo de elementos gráficos, imágenes, } \\
\text { símbolos o textos (3). }\end{array}$ \\
\hline Habilidades para la innovación y desarrollo de nuevos medios y soportes (1). \\
\hline $\begin{array}{l}\text { Habilidad para comprender y explicar la evolución del concepto de publicidad y de relaciones } \\
\text { públicas y su relación con unos determinados modelos discursivos, así como la influencia de esas } \\
\text { transformaciones en sus características actuales (1). }\end{array}$ \\
\hline $\begin{array}{l}\text { Destrezas para seleccionar y aplicar el modelo de análisis más adecuado para cada objeto de estudio } \\
\text { (2). }\end{array}$ \\
\hline Destreza en los recursos redaccionales, estilísticos, narrativos y retóricos: escritos y visuales (2). \\
\hline $\begin{array}{l}\text { Capacidad para comunicarse con fluidez y riqueza en el contexto profesoinal (de forma oral y escrita) } \\
\text { (2). }\end{array}$ \\
\hline Fundamentos de la comunicación analógica y digital para la publicidad y las relaciones públicas (1). \\
\hline Habilidad para el uso del lenguaje hipermedia (1). \\
\hline
\end{tabular}

Fuente: elaboración propia a partir de ANECA (2005, pp. 337-338).

En la materia Fundamentos y técnicas de la estrategia de la comunicación (especialmente publicitaria y de las relaciones, públicas), con $375-450$ horas de trabajo estimadas y un $40 \%$ de trabajo personal, se detallan los siguientes conocimientos:

- desarrollo de los fundamentos conceptuales de la dirección estratégica aplicados a la comunicación por objetivos para, desde un planteamiento metodológico, tomar decisiones en búsqueda de la eficacia comunicativa.

- Conocimiento del diseño y desarrollo de las estrategias y aplicaciones de las políticas de comunicación persuasiva en las organizaciones, estudiando la metodología necesaria para el estudio y la investigación del estado corporativo, evaluarlo mediante auditoría y diseñar una estrategia específica.

- Conocimiento y estudio de los procesos de comunicación y sus técnicas específicas: posicionamiento, segmentación, procedimientos de análisis y medición de la eficacia, etc.

Las destrezas, habilidades y competencias que se derivan de estos conocimientos son:

- Capacidad para la toma de decisiones estratégicas. (2)

- Capacidad para el análisis objetivo de la realidad y extracción de consideraciones válidas. (2)

- Habilidad para detectar oportunidades del mercado. (1)

- Destreza para establecer una relación de confianza, entendimiento, credibilidad y eficacia comunicativa con el anunciante.(1)

- Capacidad de programación de acciones concretas de comunicación, especialmente publicitarias y de las relaciones públicas, así como la medición de sus resultados (incluso mecanismos de retroalimentación). (3) 
- Habilidad para gestionar el tiempo, con capacidad para la organización y temporalización de las tareas. (1)

- Capacidad de análisis, de síntesis y de juicio crítico que le ayude a la conceptualización de los mensajes publicitarios así como a objetivar las tareas y relacionar las causas y los efectos. (2)

- Destrezas en las diferentes herramientas de investigación del mercado y del consumidor (pretests y post-tests) con la finalidad de obtener un conocimiento en profundidad del consumidor y su comportamiento, además de tendencias, hábitos y estilos de vida. (2)

\subsection{La creatividad y la estrategia en los perfiles profesionales de los estudios de Comunicación}

Los perfiles profesionales definidos por ANECA (2005, pp. 227-228) para la titulación de Comunicación Audiovisual son: 1. Director, guionista y realizador audiovisual; 2. productor y gestor audiovisual; 3. diseño de producción y posproducción visual y sonora e 4. investigador/a, docente y experto en estudios visuales. Por tanto, en estos perfiles no se indica ninguno directamente relacionado con la creatividad y la estrategia.

Lo mismo sucede con los perfiles profesionales definidos por ANECA (2005, pp. 191-192) para los estudios de Periodismo, al no aparecer ningún perfil profesional directamente vinculado con la creatividad y la estrategia: 1. redactor/a de información periodística en cualquier tipo de soporte; 2 . redactor/a o responsable de prensa o comunicación institucional; 3 . investigador/a, docente y/o consultor/a de comunicación y 4 . gestor/a de portales y editor/a de contenidos.

Por la variedad de especializaciones existentes en el mundo profesional y en el mercado laboral del marketing, la comunicación y la publicidad, la titulación de Publicidad y Relaciones Públicas se caracteriza por tener los perfiles profesionales más diferenciados entre sí (ANECA, 2005, pp. 271272): 1. Director/a de comunicación, investigador/a y consultor/a estratégico en publicidad y relaciones públicas; 2. Investigadores/as, Planificadores/as y Compradores de medios; 3. Creativo/a y diseñador/a y 4. Gestor/a de comunicación corporativa.

Encontramos, por tanto, dos perfiles afines a las materias de creatividad y estrategia. Los/as investigadores/as y/o consultores/as estratégicos identifican el papel específico que ha de jugar la comunicación en cada organización y en las acciones de mercadotecnia. En ambos casos, definen las estrategias de comunicación de acuerdo con los objetivos de los emisores. Planifican tanto las investigaciones ad hoc necesarias para llegar a definir los ejes fundamentales de la campaña como el desarrollo e implantación de las propias campañas que supervisan, ejecutan y/o controlan. Para ello cuentan con su conocimiento de los mercados y de las herramientas de comunicación, monitorización y control (ANECA, 2005, pp. 271-272).

En el caso del creativo/a y diseñador/a, es el profesional especializado en creatividad publicitaria en todo tipo de soportes, desde la conceptualización y visualización de la idea publicitaria hasta su adecuación e integración en los distintos medios. Se puede identificar las especialidades de: director creativo, director de arte, redactor y Webmaster -creatividad de la estructura de la Web-. La tarea de ejecutar hasta el arte final y controlar la producción física de los soportes corresponde a los expertos en producción y tráfico (ANECA, 2005, pp. 272).

Se han revisado las competencias del profesional de la publicidad y las relaciones públicas descritas por ANECA (2005, pp. 229-298) con el objeto de analizar cuáles son las más estrechamente relacionadas con cada uno de estos dos perfiles. Se muestran en la tabla 5: 
Tabla 5. Competencias del consultor estratégico y el creativo.

\begin{tabular}{|c|c|c|c|}
\hline & COMPETENCIAS & $\begin{array}{c}\text { Consultor e } \\
\text { investigador } \\
\text { estratégico }\end{array}$ & Creativo \\
\hline \multirow{10}{*}{ 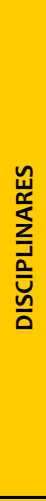 } & Conocimiento de los métodos del pensamiento creador. & $\mathrm{X}$ & $\mathrm{X}$ \\
\hline & Conocimiento de los procesos de elaboración de los mensajes publicitarios. & & $\mathrm{x}$ \\
\hline & Conocimiento teórico-práctico de los elementos, formas y procesos de los lenguajes publicitarios. & & $\mathrm{x}$ \\
\hline & $\begin{array}{l}\text { Estudio de las estrategias y procesos encaminados a la creación y realización de mensajes } \\
\text { publicitarios. }\end{array}$ & & $\mathrm{x}$ \\
\hline & Conocimiento y análisis de los procesos psicológicos básicos. & $\mathrm{x}$ & $x$ \\
\hline & Conocimiento teórico y práctico de la publicidad y las relaciones públicas. & $\mathrm{x}$ & \\
\hline & $\begin{array}{l}\text { Conocimiento de las técnicas de investigación y análisis de la naturaleza e interrelaciones entre los } \\
\text { sujetos de la comunicación publicitaria. }\end{array}$ & $\mathrm{x}$ & \\
\hline & $\begin{array}{l}\text { Estudio de los procesos de investigación e interrelaciones entre los sujetos de las actividades } \\
\text { publicitarias. }\end{array}$ & $\mathrm{X}$ & \\
\hline & Conocimiento y estudio de los procesos psicosociales, cognitivos y emocionales de la comunicación. & $\mathrm{x}$ & \\
\hline & $\begin{array}{l}\text { Conocimiento del diseño y desarrollo de las estrategias y aplicaciones de las políticas de comunicación } \\
\text { persuasiva en las instituciones y empresas públicas y privadas. }\end{array}$ & $\mathrm{X}$ & \\
\hline \multirow{5}{*}{ 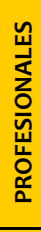 } & Capacidad y habilidad para dar forma creativa al mensaje. & & $x$ \\
\hline & Capacidad y habilidad para la creación y desarrollo de elementos gráficos. & & $x$ \\
\hline & Capacidad y habilidad para utilizar las tecnologías y técnicas comunicativas. & & $\mathrm{X}$ \\
\hline & Capacidad y habilidad para establecer el plan de comunicación. & $x$ & \\
\hline & $\begin{array}{l}\text { Capacidad relacional y de indagación para establecer una interacción fluida y competente entre el } \\
\text { anunciante y la agencia. }\end{array}$ & $x$ & \\
\hline \multirow{6}{*}{ 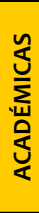 } & Conocimientos fundamentales de comunicación. & $\mathrm{X}$ & $\mathrm{X}$ \\
\hline & Capacidad para asumir el liderazgo. & $\mathrm{X}$ & $\mathrm{X}$ \\
\hline & Capacidad para entender e interpretar el entorno y adaptarse al cambio. & $\mathrm{x}$ & $x$ \\
\hline & Conocimiento del entorno económico, psicosocial, cultural y demográfico. & $x$ & $x$ \\
\hline & Capacidad para la creatividad y la innovación. & $x$ & $x$ \\
\hline & Capacidad de adaptación a los objetivos organizacionales. & $x$ & \\
\hline \multirow{9}{*}{ 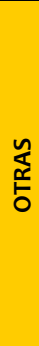 } & Capacidad de relacionarse con las personas y con el entorno sin perder su autonomía. & $\mathrm{X}$ & $\mathrm{X}$ \\
\hline & Capacidad para trabajar en equipo. & $\mathrm{x}$ & $\mathrm{x}$ \\
\hline & Capacidad de perspicacia, de ingenio y creatividad. & $\mathrm{x}$ & $\mathrm{x}$ \\
\hline & Preparación para asumir el riesgo, desarrollando la capacidad desde pensar a decidir. & $\mathrm{x}$ & $x$ \\
\hline & Capacidad de análisis, de síntesis y juicio crítico. & $x$ & $\mathrm{x}$ \\
\hline & Saber gestionar el tiempo, con habilidad para la organización y temporalización de las tareas. & $\mathrm{x}$ & $x$ \\
\hline & $\begin{array}{l}\text { Capacidad para actuar en libertad y con responsabilidad, asumiendo referentes éticos, valores y } \\
\text { principios consistentes. }\end{array}$ & $\mathrm{X}$ & $\mathrm{X}$ \\
\hline & Capacidad para la gestión económica y presupuestaria en su ámbito de actividad. & $\mathrm{x}$ & $\mathrm{x}$ \\
\hline & Capacidad para el análisis objetivo de la realidad y extracción de consideraciones válidas. & $x$ & $x$ \\
\hline
\end{tabular}

Fuente: elaboración propia a partir de ANECA (2005, pp. 229-298).

Dentro de las competencias disciplinares, consideramos que el conocimiento de los métodos del pensamiento creador $\mathrm{y}$ el conocimiento y análisis de los procesos psicológicos básicos son competencias comunes de ambos perfiles. En el caso de las competencias académicas, la figura de consultor e investigador estratégico tendría todas las propuestas por ANECA (2005). En el apartado de otras competencias ambos perfiles compartirían todas.

Dentro de perfil profesional de creativo cabe precisar la existencia de diferentes perfiles más concretos, a saber: director creativo, copy y director de arte. Tur-Viñes (2008) reclama una definición de creativo en comunicación que abandone las restricciones de lo exclusivamente publicitario y responda así a la realidad de sus funciones. Sobre el hecho de que ANECA (2005) asocie el perfil de creativo con el de diseñador, continúa la autora:

En cuanto a compartir la denominación del perfil con la de diseñador, nos parece que alienta cierta confusión, ya que, actualmente el diseñador está equiparado a un título de Formación Profesional/Bellas Artes. Con esto no queremos decir ni mucho menos que no sea necesario 
que el creativo tenga conocimientos de los programas informáticos de diseño gráfico por ordenador y de webdesign, sino que conocerlos es necesario pero no suficiente para ser creativo. Habrá que tener en cuenta que al denominar este perfil así se deja vacía de contenido la titulación de grado medio homónima. (p. 156)

A partir de estas reflexiones, Tur-Viñes (2008) propone la siguiente definición de creativo en comunicación:

Profesional especializado en la generación de ideas, en concreto en la conceptualización expresión icónica y verbal- y en la visualización -composición- de las mismas. Es el encargado de la expresión de la estrategia de comunicación concibiendo un mensaje adaptado al público buscado y realizable en un determinado medio o combinación de medios. Supervisa todo el proceso de producción y realización de la idea creativa. Por sus conocimientos sobre técnicas de generación, los creativos son idóneos como dinamizadores de cualquier proceso de innovación conducente a la creación de nuevos conceptos de productos, servicios o contenidos. (p. 15)

\subsection{La enseñanza de la creatividad y la estrategia publicitarias}

Repasamos las contribuciones realizadas por diversos autores sobre la enseñanza de las materias de creatividad y estrategia publicitarias. Muela (2012) destaca la importancia del estudio del proceso creativo aplicado a la estrategia publicitaria en la enseñanza de esta materia:

La enseñanza de la creatividad publicitaria ha priorizado el estudio de las técnicas creativas, los modelos de resolución de problemas, características psicológicas o intelectuales del sujeto, etapas del proceso o análisis del producto creativo. No obstante, además de las teorías desarrolladas sobre todo desde la psicología, también se ha procurado aplicar esos conceptos al área de la comunicación en general y a la publicitaria en particular; así, la estrategia publicitaria se explica y se practica desde la entrega de un briefing hasta la evaluación de la idea creativa en un ejercicio de role-playing. (p. 34)

El carácter transversal de la creatividad ha quedado demostrado al revisar las competencias del Grado en Publicidad y Relaciones Públicas definidas por ANECA (2005, pp. 237-296). Alegre y Roca (2012) reflexionan sobre este carácter holístico de la creatividad, como un estilo docente que se puede aplicar a cualquier materia: 
La Creatividad no es una materia aislada de los estudios de Publicidad. Si se quiere, ni siquiera tiene por qué ser (sólo) una materia. Es un enfoque, un estilo docente, una manera de entender la profesión que, si se aplica de forma holística y profunda en el desarrollo de cualquier actividad docente con gran seguridad repercutirá de forma positiva en la experiencia educativa de todas las partes implicadas. Esto supone un reto, sino una obligación, para todo el cuerpo docente universitario. (p. 18)

Marín (1989) analiza también la legislación que concierne a la enseñanza universitaria para resaltar el papel que tiene la creatividad en la formación superior:

La creatividad no es un objetivo explícito de la universidad, pero pocos discutirían que debe serlo. Más aún, está implícito en todas sus tareas.

La Ley de Reforma Universitaria de 1983 establece como funciones de la universidad en el artículo 1.2.:

a) La creación, desarrollo, transmisión y la crítica de la ciencia, de la técnica y de la cultura,

b) La preparación para el ejercicio de actividades profesionales que exijan la aplicación de conocimientos y métodos científicos o para la creación artística.

Incluso objetivos que no han sido formulados tan nítidamente y que son consustanciales a la universidad, la presuponen (...) El desarrollo y transmisión crítica de la ciencia plantea un desafío que hay que resolver con audacia creativa (...) Todas las funciones de la universidad de algún modo son, y deben ser más todavía, caminos de la creatividad, pero especialmente la investigación y el aprendizaje por descubrimiento. (pp. 35-36)

Otro enfoque sobre el estado de la cuestión de la creatividad en la enseñanza publicitaria lo ofrecen De la Torre y Violant (2003, p. 22) que, en lugar de centrarse en los alumnos, estudian al profesor. Los autores analizan las estrategias didácticas del profesorado universitario con el objetivo de describir el impacto y grado de satisfacción del alumnado ante la utilización de estrategias creativas y diferentes capaces "de hacer que los alumnos se entusiasmen por aprender".

Por su parte, Tur-Viñes (2008) describe el salto cualitativo que supuso la adaptación de la materia de creatividad del Grado en Publicidad y Relaciones Públicas al EEES: 
la creatividad seguiría siendo materia troncal pero estaría ahora aplicada al proceso de creación de mensajes comerciales, no sólo publicitarios y no sólo de relaciones públicas atendiendo a los medios. Se insistiría más en el conocimiento disciplinar y, a nivel de capacidades, se obviaría la capacidad de análisis - por ser propia de primer ciclo- y se incidiría en la capacidad de generar, plantear y defender, de forma coherente y pertinente, estrategias de comunicación comercial. (pp. 158-159)

Encontramos ejemplos de la aplicación del aprendizaje cooperativo y de nuevas técnicas docentes en asignaturas relacionadas con la materia de creatividad publicitaria, como es el caso de Estrategias Creativas en Publicidad en el Grado en Publicidad y Relaciones Públicas de la Universidad Rey Juan Carlos (Muela, 2012) o Pensamiento Creativo en el grado en Comunicación de la Universitat Oberta de Catalunya (Sivera-Bello, 2016). En el primer caso, el proyecto de innovación docente demostró que el trabajo cooperativo a partir de la técnica puzzle ayuda a facilitar el aprendizaje e incrementa el conocimiento de la realidad profesional, de sus agentes y protagonistas respecto a la creatividad publicitaria (Muela, 2012).

Pasando a revisar la enseñanza de la estrategia en el Grado, esta disciplina se encuentra limitada a los estudios de Publicidad y Relaciones Públicas. No encontramos en el Libro Blanco de ANECA (2005) en las otras dos titulaciones de Comunicación ninguna alusión a la formación en este ámbito, lo que se traduce en la ausencia clara de materias con estos contenidos (Torres, 2010).

A pesar de la saturación de la oferta de estudios de comunicación en nuestro país, los contenidos o asignaturas relacionados con la disciplina estratégica resultan escasos. La tendencia a la reproducción de los modelos anteriores ha supuesto que el proceso de adaptación no haya resuelto esta situación. Lo que resulta claro es que las competencias y capacidades del alumno de comunicación en cuestiones estratégicas son altamente valoradas en todos los nuevos planes de estudio. Esto se traduce en una inflación en el uso del término estrategia y, más concretamente, al relacionarlo con el ámbito publicitario. (p. 307)

Esta realidad puede justificarse con la importancia objetiva que tiene la formación estratégica en los estudios a los que nos estamos refiriendo concretamente, desde dos puntos de vista: el de la planificación estratégica y el de la creación estratégica, precisamente los dos ejes básicos para el desarrollo de la actividad publicitaria y de las relaciones públicas, si bien es cierto que esta afirmación depende de la perspectiva que adoptemos al hablar de estrategia. (p. 312) 
Desde este punto de vista, las titulaciones en el ámbito de la comunicación en España se caracterizan por la disparidad en la implantación de los estudios de estrategia (Torres, 2010, p. 311). El término estrategia es aplicado por diversos autores, estudiosos o profesionales de la publicidad a casi todas las actividades que pueden desarrollarse en este campo. Así, se habla de "estrategia general de la publicidad", de "estrategia de medios", "estrategia creativa", "estrategia del mensaje", "copy strategy", etc. Esto supone que si nos referimos a estrategia en el ámbito publicitario exista cierta confusión sobre qué elementos están incluidos en ella. De esta forma, nos encontramos con que, además de la inflación del término, en publicidad es donde es utilizado de una manera más subjetiva (Pérez-González, 1989).

Mientras que una parte importante de autores determina que la estrategia publicitaria sería la etapa de elaboración del "qué" o contenido del mensaje de la comunicación publicitaria, de acuerdo con los objetivos asignados, para otro grupo en la estrategia publicitaria la elaboración del "qué" debería situarse en la estrategia creativa (Davara, 1994, p. 189), por lo que se reservarían la expresión "estrategia publicitaria" para definir la selección de métodos de trabajo; es decir, un paso previo a la acción (García-Uceda, 2001).

De estos conocimientos se extraen varios conceptos básicos en la teoría estratégica: la toma de decisiones, las estrategias de creación de mensajes y el diseño de estrategias específicas. Se trata, además, de los elementos en los que la teoría estratégica ha demostrado un mayor desarrollo. En primer lugar, la relación entre la toma de decisiones y la prospectiva; en segundo lugar, los modelos estratégicos del ámbito publicitario que evolucionaron desde las estrategias basadas en el producto Unique Selling Proposition de Reeves-, hasta las de posicionamiento e imagen de marca actuales (Kapferer, 1997); por último, nos situamos en el proceso de planificación estratégica publicitaria como forma de elaboración de los planes comunicativos de las instituciones (Pérez-González, 1989).

Torres (2010: 315) concluye que la amplia oferta educativa en comunicación no recoge, sin embargo, las necesidades formativas en el ámbito de la disciplina estratégica. No obstante, lo que sí resulta claro es la orientación profesionalizante de la enseñanza de estrategias de comunicación.

\subsection{La investigación sobre la enseñanza de la creatividad publicitaria}

Por último, señalamos la investigación sobre la enseñanza de la creatividad en la universidad realizada por Alegre y Roca (2012) por la extensa revisión de bibliografía que realizan, dividiendo los estudios en dos grandes grupos: análisis y metodología. En el análisis se incluyen todos los artículos que estudian el estado de la enseñanza de la creatividad, normalmente, usando la metodología cuantitativa mediante cuestionarios a profesores. El grupo de metodología contiene todos aquellos artículos que describen el desarrollo de la asignatura de creatividad publicitaria, normalmente a partir de la descripción de la propia experiencia docente del autor. Con esta revisión, los autores llegan a las siguientes conclusiones (Alegre y Roca, 2012, p. 75) sobre la enseñanza de la creatividad publicitaria en la universidad:

- La enseñanza de la creatividad es holística. El profesor que pretenda enseñar creatividad publicitaria debe tener en cuenta que apelará a muchos factores del desarrollo de su alumno, no solo a nivel intelectual o profesional, sino también personal. El objetivo último de la enseñanza no es la transmisión de conocimientos, sino la formación de una actitud.

- La enseñanza de la creatividad requiere implicación. Cuando se enseña creatividad publicitaria, profesor y alumno comparten mucho más que el espacio del aula. El profesor es el encargado de que sus estudiantes se autoafirmen como personas creadoras y creativas. Esto se logra manteniendo, en la medida de lo posible, una relación estrecha con los alumnos y generando un clima de seguridad y confianza mutua en la clase. 
- La enseñanza de la creatividad requiere creatividad. Aunque suene a tautología, parece inconcebible que una asignatura de creatividad tenga éxito si quien la imparte no está dispuesto a utilizar su imaginación y sus propios recursos para organizarla.

- La enseñanza de la creatividad es real. La cuestión no es si la creatividad se puede enseñar o no. La creatividad publicitaria se está enseñando con éxito cada día en todo el mundo. La cuestión es seguir mejorando los modelos, actitudes, espacios y técnicas para enseñarla.

El estudio de Alegre y Rosa (2012) es una adaptación para el territorio español de la investigación de Stuhlfaut y Berman (2009) en Estados Unidos. En su investigación, Stuhlafaut y Berman seleccionaron una muestra de 44 universidades de las 145 -esto es, el 30\%- existentes en Estados Unidos en las que se imparten programas de publicidad y relaciones públicas. Estas se seleccionaron de tres listas que aparecen en A Century of Advertising Education (Ross y Richards, 2008). Examinaron la página web de las universidades para detectar las asignaturas de estrategia creativa creative strategy-, incluyendo también las de dirección de arte -advertising art-, redacción creativa copywriting- y dejando fuera asignaturas de elaboración de campañas -campaigns-, creatividad general -general creativity- y book -portfolio development-. Los autores entienden que la dirección de arte publicitaria y la redacción creativa son ramas de la estrategia creativa.

Alegre y Roca (2012) identificaron 286 asignaturas de creatividad publicitaria de los Grados en Publicidad y Relaciones Públicas en España. A partir de los contenidos y objetivos observados en las asignaturas analizadas, establecen una serie de descriptores comunes (Alegre y Roca, 2012, p. 90) ${ }^{2}$, identificando bloques que son fieles a la idiosincrasia del departamento creativo en la agencia de publicidad:

- Estrategia Creativa. En este grupo tienen cabida todas aquellas asignaturas en las cuales los alumnos ponen en práctica los conocimientos de redacción creativa para el desarrollo de campañas integrales de publicidad. Los objetivos suelen incluir el desarrollo del pensamiento estratégico y una comprensión global del proceso creativo publicitario: desde el briefing hasta la implementación en los medios. Estas asignaturas ofrecen una visión global de la creatividad publicitaria, de modo que para desarrollar las campañas de comunicación -que suelen ser requeridas como método de evaluación- los alumnos deben comprender en profundidad la marca, el target, el mercado, etc.

- Redacción Creativa. Son aquellas asignaturas en las cuales los alumnos desarrollan las habilidades y criterios necesarios para redactar de forma creativa en el entorno publicitario. Es habitual que estas asignaturas vertebren su temario según los diferentes tipos de medios en los cuales un redactor creativo debe desenvolverse y las características de estos. Aunque en menor medida, desarrollar las habilidades creativas a nivel global suele ser otro de los objetivos comunes de estas asignaturas.

- Dirección de Arte. Forman parte de este grupo las asignaturas destinadas al estudio y la comprensión del lenguaje visual y su uso en la publicidad. Aunque pueden incluir formación en programas informáticos de edición de imagen, estas asignaturas están más orientadas hacia el conocimiento de los elementos formales de la comunicación a través de la imagen.

- Creatividad Publicitaria. Estas asignaturas de creatividad general se centran en tres aspecto principales: a) El desarrollo de las habilidades creativas -que incluye aprendizaje de técnicas

\footnotetext{
${ }^{2}$ Cabe apuntar que en el proyecto de investigación de Alegre y Roca (2012) no se incluyen las asignaturas de diseño gráfico. Pese a que estas se recogieron durante la toma de la muestras, a fin de acotar el estudio, los autores optaron por analizar únicamente las asignaturas de dirección de arte. Pese a que resulta innegable que la creatividad es un factor crucial en el desarrollo del diseño, existe un debate abierto sobre el papel exacto que ésta juega (Dineen y Collins, 2004, p. 1). A fin de no entrar en este debate, los autores decidieron ceñirse a las cuatro asignaturas descritas. Es preciso destacar, no obstante, que las asignaturas de dirección de arte, diseño gráfico y afines, tienen un gran peso en los planes de estudios.
} 
creativas-. b) La comprensión teórica del fenómeno de la Creatividad -proceso creativo, persona creativa, etc.- en general y de su aplicación en comunicación y publicidad. c) El desarrollo de una actitud creativa más allá de la profesión publicitaria.

Una de las conclusiones a las que llegaron fue que existía una gran diversidad en los nombres de las asignaturas, con 17 nombres para las asignaturas de redacción, 21 para las de arte y 37 para las de estrategia creativa.

\subsection{La asignatura Estrategia Creativa y Conceptualización en el Grado en Publicidad y Relaciones Públicas de la Universidad de Alicante}

En este apartado nos detenemos en la asignatura Estrategia Creativa y Conceptualización del Grado en Publicidad y Relaciones Públicas de la Universidad de Alicante, como ejemplo de asignatura del ámbito que nos ocupa y a partir de la cual planteamos la investigación que se describe a continuación. Esta materia, obligatoria de tercer curso, pertenece al módulo que lleva por título Creatividad en comunicación publicitaria y de las relaciones públicas. El descriptor del módulo indica que ${ }^{3}$ :

Abarca, primero, el conocimiento del concepto de creatividad, las teorías explicativas

existentes así como el tipo de pensamiento que favorece las producciones creativas; segundo,

el análisis de la publicidad como forma de comunicación informativo-persuasiva y sus

implicaciones en el ámbito del lenguaje; en tercer lugar, según su ubicación en el plan de

estudios, se aborda el conocimiento del proceso estratégico que determina las decisiones

creativas, así como los principales géneros y recursos que conducen a la conceptualización,

entendida como la expresión del mensaje, y la adaptación de los conceptos a los diferentes

medios de comunicación. En cuanto a las asignaturas optativas se profundiza en el

conocimiento y desarrollo de habilidades necesarias para hacer más eficaz la comunicación a

través de su correcta redacción, visualización y composición, así como del uso sinérgico de

las formas emergentes de comunicación no convencional.

Además de Estrategia creativa y conceptualización, las asignaturas incluidas dentro de este módulo, con 6 créditos ECTS cada una de ellas, son:

- Fundamentos de la creatividad (obligatoria de segundo curso).

- Lenguaje publicitario (obligatoria de segundo curso).

- Elaboración de textos publicitarios (obligatoria de tercer curso).

- Dirección de arte (optativa en cuarto curso).

- Nueva formas publicitarias (optativa en cuarto curso).

- Cartel publicitario (optativa en cuarto curso).

\footnotetext{
${ }^{3}$ Extraído del plan de estudios del Grado: http://bit.ly/grado-publicidad-ua.
} 
Estas asignaturas están organizadas dentro del plan de estudios de manera que el alumno se sumerge en el proceso creativo desde un nivel macro -estudiando el concepto de creatividad y los mecanismos de creación- a un nivel micro -en asignaturas como Lenguaje Publicitario, Elaboración de Textos Publicitarios y Dirección de Arte la creatividad se aplica a la expresión verbal y visual del mensaje persuasivo-, pasando por la asignatura Estrategia Creativa y Conceptualización, que es el nexo de unión entre ambos niveles, al profundizar en la conceptualización de la creatividad en el proceso de la estrategia de comunicación persuasiva. Este proceso estratégico que determina las decisiones creativas es previo a la creación de la parte icónica y verbal del mensaje.

Las asignaturas optativas Nuevas Formas Publicitarias y Cartel Publicitario introducen la aplicación de la creatividad en el uso de las formas emergentes de comunicación no convencional y profundizan en la componente visual del mensaje, respectivamente. La asignatura que guarda una vinculación más estrecha con Estrategia Creativa y Conceptualización es Fundamentos de la Creatividad.

Lo propio y específico de la asignatura Estrategia creativa y conceptualización en relación al campo científico al que pertenece es la combinación de la creatividad con la estrategia, lo que la hace única en el plan de estudios del Grado en Publicidad y Relaciones Públicas de la Universidad de Alicante. El estudio de la creatividad desde un enfoque estratégico -la comunicación persuasiva está al servicio de unos objetivos que se pretenden alcanzar dando con la solución más conveniente a un problema de comunicación- y de la estrategia desde un enfoque creativo -si una de la premisas de cualquier estrategia es el hecho de contar con una serie de recursos disponibles, a veces limitados, es precisamente por esta causa por la que hay que agudizar el ingenio y la capacidad de innovación- es lo que hace de Estrategia Creativa y Conceptualización una asignatura necesaria en la formación del futuro profesional de la publicidad y las relaciones públicas.

\section{Método}

\subsection{Estrategias metodológicas}

El objetivo principal del estudio que se presenta es analizar la presencia de asignaturas de creatividad y estrategia en los grados en Comunicación en España. La investigación parte de las aportaciones analizadas en la revisión bibliográfica y presentadas en la introducción, en especial de la de Alegre y Roca (2012), y se propone como objetivos específicos los siguientes:

- identificar asignaturas similares a Estrategia Creativa y Conceptualización en los Grados en Comunicación en España.

- revisar las nomenclaturas de las asignaturas de estrategia y creatividad en los Grados en Comunicación en España.

- estudiar el reparto de asignaturas de estrategia y creatividad en los Grados en Comunicación en España entre las tres titulaciones, a saber: Comunicación Audiovisual, Periodismo y Publicidad y Relaciones Públicas.

La hipótesis principal destaca la poca variedad de asignaturas existentes en los Grados en Comunicación que combinan el estudio de la creatividad y la estrategia. Por su parte, las asignaturas de creatividad son más populares que las de estrategia y es en las titulaciones en Publicidad y Relaciones Públicas en las que ambas tienen una mayor presencia. Además, las asignaturas de creatividad y/o estrategia en los Grados en Comunicación se caracterizan por la diversidad de nomenclaturas. 


\subsection{Población y muestra}

Para alcanzar los objetivos y revisar la validez de las hipótesis, el estudio parte de la revisión de la oferta de estudios en Comunicación en España, con un análisis a partir de los planes de estudio de 125 titulaciones, procedentes de 61 centros. Estos 125 grados representan el 13,5\% si los ponemos en relación con el total de grados ofertados en España en la rama de Ciencias Sociales y Jurídicas, 927 (Ministerio de Educación, Cultura y Deporte, 2016, p. 7). De los 125 grados analizados, 62 grados proceden de universidades públicas y 63 de instituciones privadas. En total, la muestra de titulaciones procede de 32 universidades públicas y 29 centros privados.

En la tabla 6 pueden verse las 61 universidades de la muestra, la comunidad autónoma a la que pertenecen, la titularidad, la facultad a la que se adscriben los títulos de Comunicación y el número de grados ofertados. 
RLCS, Revista Latina de Comunicación Social, 77, 143-178

[Investigación] DOI: 10.4185/RLCS-2020-1453 | ISSN 1138-5820 | Año 2020

Tabla 6. Titulaciones en Comunicación en centros universitarios españoles.

\begin{tabular}{|c|c|c|c|c|}
\hline $\begin{array}{l}\text { Comunidad } \\
\text { Autónoma }\end{array}$ & Universidad & Tipo & Facultad / Centro & $\begin{array}{c}\text { Número de } \\
\text { grados }\end{array}$ \\
\hline Comunidad de Madrid & Universidad Autónoma de Madrid & Pública & Fac. Lenguas Modernas, Cultura y Comunicación & 1 \\
\hline Comunidad de Madrid & Universidad Carlos III & Pública & Fac. Humanides, Comunicación y Documentación & 2 \\
\hline Castilla La Mancha & Universidad Castilla La Mancha & Pública & Fac. Periodismo & 1 \\
\hline Comunidad de Madrid & Universidad Complutense de Madrid & Pública & Fac. Ciencias de la Información & 3 \\
\hline Comunidad de Madrid & Universidad de Alcalá & Pública & Fac. Filosofía y Letras & 1 \\
\hline Comunidad Valenciana & Universidad de Alicante & Pública & Fac. Ciencias Económicas y Empresariales & 1 \\
\hline Andalucía & Universidad de Cádiz & Pública & Fac. Ciencias Sociales y de la Comunicación & 1 \\
\hline Extremadura & Universidad de Extremadura & Pública & Fac. Ciencias de la Documentación y la Comunicación & 1 \\
\hline Andalucía & Universidad de Granada & Pública & Fac. Comunicación y Documentación & 1 \\
\hline Islas Canarias & Universidad de La Laguna & Pública & Fac. Ciencias Políticas, Sociales y de la Comunicación & 1 \\
\hline Andalucía & Universidad de Málaga & Pública & Fac. Ciencias de la Comunicación & 3 \\
\hline Región de Murcia & Universidad de Murcia & Pública & Fac. Comunicación y Documentación & 3 \\
\hline Castilla y León & Universidad de Salamanca & Pública & Fac. Ciencias Sociales & 1 \\
\hline Andalucía & Universidad de Sevilla & Pública & Fac. Comunicación & 3 \\
\hline Castilla y León & Universidad de Valladolid & Pública & Fac. Ciencias Sociales, Jurídicas y de la Comunicación & 2 \\
\hline Galicia & Universidad de Vigo & Pública & Fac. Ciencias Sociales y de la Comunicación & 2 \\
\hline Aragón & Universidad de Zaragoza & Pública & Fac. Filosofía y Letras & 1 \\
\hline País Vasco & Universidad del País Vasco & Pública & Fac. Ciencias Sociales y de la Comunicación & 3 \\
\hline Comunidad Valenciana & Universidad Miguel Hernández & Pública & Fac. Ciencias Sociales y Jurídicas & 2 \\
\hline Comunidad de Madrid & Universidad Rey Juan Carlos & Pública & Fac. Ciencias de la Comunicación & 4 \\
\hline Galicia & Universidade da Coruña & Pública & Fac. Ciencias de la Comunicación & 1 \\
\hline Galicia & Universidade de Santiago de Compostela & Pública & Fac. Ciencias de la Comunicación & 2 \\
\hline Cataluña & Universitat Autònoma de Barcelona & Pública & Fac. Ciencias de la Comunicación & 2 \\
\hline Cataluña & Universitat de Barcelona & Pública & Fac. Biblioteconomía y Documentación & 3 \\
\hline Cataluña & Universitat de Girona & Pública & Fac. Turismo & 2 \\
\hline Islas Baleares & Universitat de les Illes Balears & Pública & Centro de Enseñanza Superior Alberta Giménez & 2 \\
\hline Cataluña & Universitat de Lleida & Pública & Fac. Letras & 1 \\
\hline Comunidad Valenciana & Universitat de València & Pública & Fac. Filología, Traducción y Comunicación & 2 \\
\hline Comunidad Valenciana & Universitat Jaume I & Pública & Fac. Ciencias Humanas y Sociales & 3 \\
\hline Comunidad Valenciana & Universitat Politècnica de València & Pública & Escuela Politècnica Superior de Gandia & 1 \\
\hline Cataluña & Universitat Pompeu Fabra (UPF) & Pública & Fac. Comunicación & 3 \\
\hline Cataluña & Universitat Rovira i Virgili & Pública & Fac. Letras & 3 \\
\hline Cataluña & Bau Centro Universitario de Diseño de Barcelona & Privada & & 1 \\
\hline Comunidad de Madrid & Centro de Estudios Superiores Felipe II & Privada & & 1 \\
\hline Cantabria & CESINE & Privada & Escuela de Negocios y Comunicación & 1 \\
\hline Comunidad Valenciana & Instituto Mediterráneo de Estudios de Protocolo & Privada & & 1 \\
\hline Cataluña & Escuela Universitaria Formatic Barcelona & Privada & - & 1 \\
\hline Comunidad de Madrid & ESIC, BUSINESS\&MARKETING SCHOOL (Madrid) & Privada & & 1 \\
\hline Comunidad Valenciana & ESIC, BUSINESS\&MARKETING SCHOOL (Valencia) & Privada & & 1 \\
\hline Cataluña & EUNCET Business School - UPC & Privada & - & 1 \\
\hline Cataluña & L'Idem Barcelona & Privada & - & 1 \\
\hline País Vasco & Mondragon Unibertsitatea (UdM) & Privada & & 1 \\
\hline Comunidad de Madrid & UDIMA-Universidad a Distancia de Madrid & Privada & Fac. Ciencias Sociales y Humanidades & 2 \\
\hline La Rioja & UNIR Universidad Internacional La Rioja & Privada & Fac. Ciencias Jurídicas, Sociales y Humanidades & 1 \\
\hline Comunidad de Madrid & Universidad Camilo José Cela & Privada & Fac. Comunicación & 2 \\
\hline Comunidad Valenciana & Universidad Cardenal Herrera-CEU & Privada & Fac. Humanidades y Ciencias de la Comunicación & 3 \\
\hline Región de Murcia & Universidad Católica San Antonio & Privada & Fac. Ciencias Sociales y de la Comunicación & 4 \\
\hline Comunidad de Madrid & Universidad CEU San Pablo & Privada & Fac. Humanidades y Ciencias de la Comunicación & 4 \\
\hline País Vasco & Universidad de Deusto & Privada & Fac. Ciencias Sociales y Humanas & 1 \\
\hline Navarra & Universidad de Navarra & Privada & Fac. Comunicación & 3 \\
\hline Islas Canarias & Universidad Europea Canarias & Privada & Fac. Ciencias Sociales & 1 \\
\hline Castilla y León & Universidad Europea Miguel de Cervantes (UEMC) & Privada & Fac. Ciencias Sociales & 2 \\
\hline Comunidad de Madrid & Universidad Francisco de Vitoria & Privada & Fac. Ciencias de la Comunicación & 3 \\
\hline Cataluña & Universitat Internacional de Catalunya & Privada & Fac. Ciencias de la Comunicación & 3 \\
\hline Comunidad de Madrid & Universidad Nebrija & Privada & Fac. Ciencias de la Comunicación & 4 \\
\hline Castilla y León & Universidad Pontificia Salamanca & Privada & Fac. Comunicación & 4 \\
\hline Aragón & Universidad San Jorge & Privada & Fac. Comunicación y Ciencias Sociales & 4 \\
\hline Cataluña & Universidad Abat Oliba CEU & Privada & Fac. Ciencias Sociales & 2 \\
\hline Cataluña & Universitat de Vic & Privada & Fac. Empresa y Comunicación & 4 \\
\hline Cataluña & Universitat Oberta de Catalunya & Privada & Estudios de Ciencias de la Información y de la Comunicación & 1 \\
\hline \multirow[t]{2}{*}{ Cataluña } & Universitat Ramon Llull & Privada & Fac. Comunicación y Relaciones Internacionales Blanquerna & 5 \\
\hline & & & & 125 \\
\hline
\end{tabular}

Fuente: elaboración propia a partir de datos de las páginas web de las universidades.

En función del número de titulaciones en Comunicación, sólo hay un centro que ofrece cinco titulaciones en este ámbito: la universidad privada Universitat Ramon Llull. Son 7 los centros que ofrecen 4 titulaciones -de ellos, 6 son privados-, 13 universidades cuentan con 3 titulaciones y 2 
titulaciones y son 27 (44,3\%) los centros que ofertan un único grado en Comunicación. Por tanto, la media de titulaciones por centro es de 2 , si bien la moda se encuentra en una única titulación. Se incluye este desglose en la tabla 7 y en la figura 1.

Tabla 7. Número de titulaciones por Universidad.

\begin{tabular}{|l|c|c|r|c|}
\hline \multirow{2}{*}{$\begin{array}{c}\text { Número de } \\
\text { titulaciones }\end{array}$} & \multicolumn{2}{|c|}{ Centros } & Total & $\begin{array}{c}\text { Total } \\
\text { titulaciones }\end{array}$ \\
\cline { 2 - 3 } Cinco & Públicos & Privados & centros & tul \\
\hline Cuatro & 0 & 1 & 7 & 5 \\
\hline Tres & 1 & 6 & 13 & 28 \\
\hline Dos & 9 & 4 & 13 & 26 \\
\hline Uno & 9 & 4 & 27 & 27 \\
\hline
\end{tabular}

Fuente: elaboración propia a partir de datos de las páginas web de las universidades.

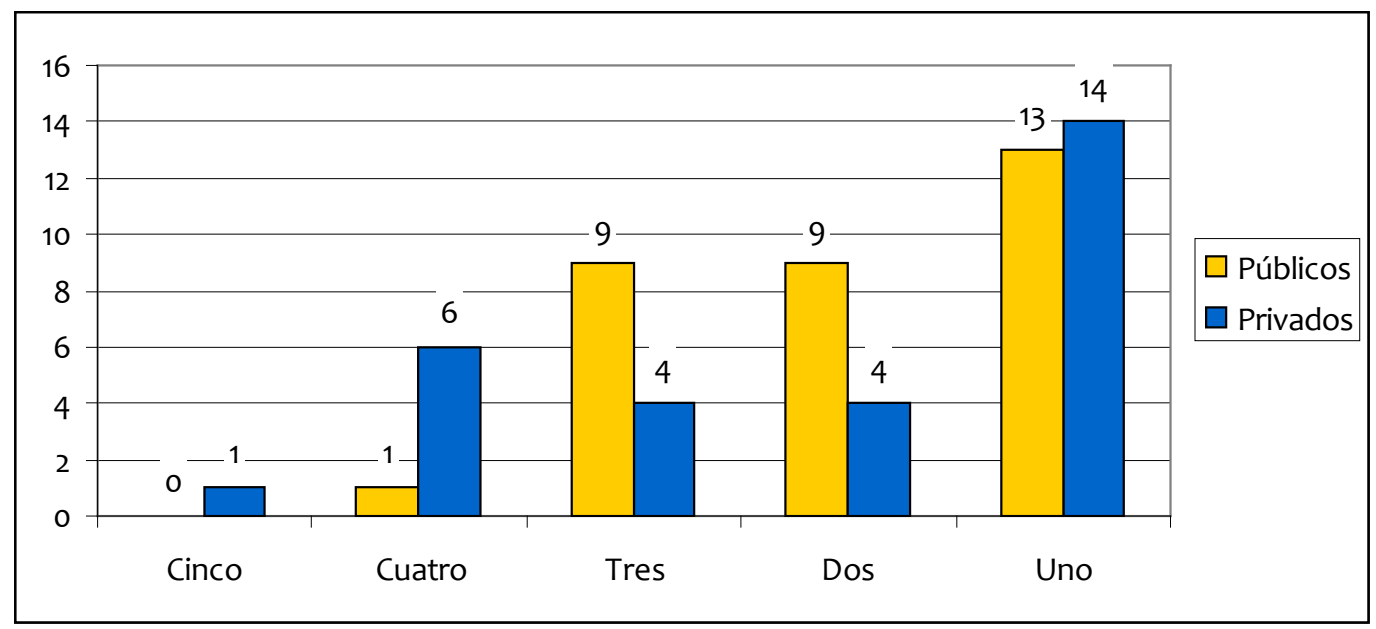

Figura 1: número de centros en función del número de titulaciones en Comunicación.

Fuente: elaboración propia a partir de datos de las páginas web de las universidades.

Las titulaciones identificadas se organizan por categorías, a partir de los tres Grados en Comunicación descritos por ANECA (2005): Comunicación Audiovisual, Periodismo y Publicidad y Relaciones Públicas. Además, se añaden dos categorías más, para abarcar los Grados en Comunicación así como otras titulaciones afines.

Por categoría, como puede verse en la tabla 8, de los 125 grados en Comunicación identificados en los 61 centros, 14 corresponden a Comunicación (11,2\%), 37 a Comunicación Audiovisual (29,6\%), 33 titulaciones son de Periodismo (26,4\%), 32 son de Publicidad y Relaciones Públicas $(25,6 \%)$ y 9 de otras titulaciones afines (7,2\%) -relacionadas con el protocolo, el diseño, la organización de eventos, la comunicación digital y/o el cine-. De esta manera, las tres ramas dentro de las Ciencias de la Comunicación identificadas en España desde 1991 son las que representan la mayor oferta de estudios de Comunicación. 
A partir de este desglose, se lleva a cabo un estudio de las asignaturas incluidas en los planes de estudios relacionadas con la asignatura Estrategia Creativa y Conceptualización. El análisis se ha basado en la nomenclatura de las asignaturas, estableciendo tres niveles de relación:

- Nivel 1. Asignaturas similares a Estrategia Creativa y Conceptualización. Se identifican por la presencia en la nomenclatura de dos términos coincidentes relacionados con la estrategia, la creatividad y/o la conceptualización.

- Nivel 2. Asignaturas en cuyo nombre se incluyen sustantivos como estrategia, creatividad o conceptualización. Se incorporan también aquellas asignaturas cuyo título se refiere a la innovación, la ideación, la creación y/o a la generación de ideas. Cabe precisar que se han descartado algunas asignaturas en cuyo nombre se incluía el sustantivo estrategia pero relacionado a otros ámbitos - como estrategia de precios o estrategia de programación-. En cualquier caso, el total de estas asignaturas descartadas ha sido inferior a diez.

- Nivel 3. Asignaturas en cuyo nombre se incluye un adjetivo relacionado con la estrategia creativa y la conceptualización: estratégico, creativo, conceptual.

En total, se identifican 202 asignaturas: 9 asignaturas en el nivel 1, 123 asignaturas de nivel 2 y 70 asignaturas de nivel 3.

\subsection{Instrumentos de recogida de información y procedimiento}

La recopilación de la información se realizó entre mayo y julio de 2017 a través de las páginas web de las universidades, accediendo a los planes de estudio de las titulaciones de la muestra para identificar las asignaturas relacionadas a partir de los niveles anteriormente indicados. Por tanto, la recogida de la información se llevó a cabo mediante acceso a Internet y a partir del análisis de la nomenclatura de las asignaturas incluidas en los 125 Grados.

\section{Resultados}

Las 202 asignaturas identificadas proceden de 79 titulaciones -es decir, del 63,2\% de las titulaciones analizadas, con una media de 2,6 asignaturas por titulación-, mientras que son 46 los Grados en los que no aparecen asignaturas con nombres relacionados con Estrategia Creativa y Conceptualización, ofertados por 34 centros diferentes. Ninguna de estas 46 titulaciones sin asignaturas vinculadas con la estrategia creativa y la conceptualización corresponde a la categoría publicidad y relaciones públicas: 16 son de comunicación audiovisual, 21 de periodismo, 4 de comunicación y 5 de otras, como muestra la tabla 8.

Tabla 8. Titulaciones sin asignaturas relacionadas.

\begin{tabular}{|l|r|}
\hline \multicolumn{1}{|c|}{ Categoría } & Número de titulaciones \\
\hline Comunicación Audiovisual & 16 \\
\hline Periodismo & 21 \\
\hline Comunicación & 4 \\
\hline Otras & 5 \\
\hline
\end{tabular}

Fuente: elaboración propia a partir de datos de las páginas web de las universidades.

Por el contrario, el desglose de las 79 titulaciones con asignaturas vinculadas con la estrategia creativa y la conceptualización es el que muestra la tabla 9. 
RLCS, Revista Latina de Comunicación Social, 77, 143-178

[Investigación] DOI: 10.4185/RLCS-2020-1453 | ISSN 1138-5820 | Año 2020

Tabla 9. Titulaciones con asignaturas vinculadas con la estrategia creativa y la conceptualización.

\begin{tabular}{|c|c|c|c|c|c|c|c|c|c|}
\hline \multirow{3}{*}{$\begin{array}{l}\text { Categoría de titulación } \\
\text { Comunicación Audiovisual }\end{array}$} & \multirow{3}{*}{\begin{tabular}{|r|}
$N^{\circ}$ titulaciones \\
21 \\
\end{tabular}} & \multicolumn{8}{|c|}{ Asignaturas } \\
\hline & & \multicolumn{2}{|c|}{ Nivel 1} & \multicolumn{2}{|c|}{ Nivel 2} & \multicolumn{2}{|c|}{ Nivel 3} & \multicolumn{2}{|c|}{ Total niveles } \\
\hline & & 0 & $0,0 \%$ & 30 & $22,9 \%$ & 3 & $4,8 \%$ & 33 & $16,3 \%$ \\
\hline Periodismo & 12 & 0 & $0,0 \%$ & 9 & $6,9 \%$ & 4 & $6,5 \%$ & 13 & $6,4 \%$ \\
\hline Publicidad y Relaciones Públicas & 32 & 9 & $100,0 \%$ & 74 & $56,5 \%$ & 44 & $71,0 \%$ & 127 & $62,9 \%$ \\
\hline Comunicación & 10 & 0 & $0,0 \%$ & 15 & $11,5 \%$ & 10 & $16,1 \%$ & 25 & $12,4 \%$ \\
\hline Otras & 4 & 0 & $0,0 \%$ & 3 & $2,3 \%$ & 1 & $1,6 \%$ & 4 & $2,0 \%$ \\
\hline Total & 79 & 9 & $100,0 \%$ & 131 & $100,0 \%$ & 62 & $100,0 \%$ & 202 & $100 \%$ \\
\hline
\end{tabular}

Fuente: elaboración propia a partir de datos de las páginas web de las universidades.

En total, el $62,9 \%$ de las asignaturas identificadas se encuentran en los Grados en Publicidad y Relaciones Públicas. Las 32 titulaciones de Publicidad y Relaciones de la muestra tienen asignaturas relacionadas en alguno de los tres niveles identificados, lo que supone una media de 4 asignaturas por Grado. Además, las 9 asignaturas de nivel 1 proceden de los Grados en Publicidad y Relaciones Públicas. El $60,2 \%$ de las asignaturas del nivel 2 y el $62,9 \%$ de las asignaturas del nivel 3 se encuentran en esta categoría de Grados. En las tablas 10 a 14 se muestra el desglose de asignaturas relacionadas con la estrategia creativa y la conceptualización para cada categoría de titulaciones:

Tabla 10. Grados en Comunicación Audiovisual en cuyos planes de estudio se incluyen asignaturas relacionadas.

\begin{tabular}{|c|c|c|c|c|c|}
\hline \multirow[b]{2}{*}{ Universidad } & \multirow[b]{2}{*}{ Categoría: Comunicación Audiovisual } & \multicolumn{4}{|c|}{ Asignaturas relacionadas } \\
\hline & & Nivel 1 & Nivel 2 & Nivel 3 & Total \\
\hline Centro de Estudios Superiores Felipe II & Comunicación Audiovisual & 0 & 1 & 0 & \\
\hline Universidad Carlos III & Comunicación Audiovisual & 0 & 1 & 0 & \\
\hline Universidad Católica San Antonio & Comunicación Audiovisual & 0 & 1 & 0 & \\
\hline \begin{tabular}{|l|} 
Universidad de Alcalá \\
\end{tabular} & Comunicación Audiovisual & 0 & 0 & 1 & \\
\hline Universidad de Extremadura & Comunicación Audiovisual & 0 & 1 & 0 & \\
\hline Universidad de Málaga & Comunicación Audiovisual & 0 & 1 & 0 & \\
\hline Universidad de Murcia & Comunicación Audiovisual & 0 & 1 & 0 & \\
\hline Universidad de Sevilla & Comunicación Audiovisual & 0 & 0 & 1 & \\
\hline \begin{tabular}{|l} 
Universidad de Vigo \\
\end{tabular} & Comunicación Audiovisual & 0 & 1 & o & \\
\hline Universidad del País Vasco & Comunicación Audiovisual & 0 & 3 & 0 & \\
\hline Universidad Francisco de Vitoria & Comunicación Audiovisual & 0 & 1 & 0 & \\
\hline Universidad Nebrija & Comunicación Audiovisual & 0 & 1 & o & \\
\hline Universidad Rey Juan Carlos & Comunicación Audiovisual & 0 & 2 & o & 2 \\
\hline Universidade da Coruña & Comunicación Audiovisual & 0 & 1 & 0 & \\
\hline \begin{tabular}{|l|} 
Universidade de Santiago de Compostela \\
\end{tabular} & Comunicación Audiovisual & 0 & 1 & o & \\
\hline Universitat de Vic & Comunicación Audiovisual & 0 & 1 & 0 & \\
\hline Universitat Jaume I & Comunicación Audiovisual & 0 & 1 & 0 & \\
\hline Universitat Politècnica de València & Comunicación Audiovisual & 0 & 3 & o & 3 \\
\hline Universitat Pompeu Fabra (UPF) & Comunicación Audiovisual & 0 & 5 & o & 5 \\
\hline Universitat Rovira i Virgili & Comunicación Audiovisual & 0 & 0 & 1 & \\
\hline Universitat de Lleida & Comunicación y Periodismo Audiovisuales & 0 & 4 & o & 4 \\
\hline & & 0 & 30 & 3 & 33 \\
\hline
\end{tabular}

Fuente: elaboración propia a partir de datos de las páginas web de las universidades. 
RLCS, Revista Latina de Comunicación Social, 77, 143-178

[Investigación] DOI: 10.4185/RLCS-2020-1453 | ISSN 1138-5820 | Año 2020

Tabla 11. Grados en Periodismo en cuyos planes de estudio se incluyen asignaturas relacionadas.

\begin{tabular}{|c|c|c|c|c|c|}
\hline \multirow[b]{2}{*}{ Universidad } & \multirow[b]{2}{*}{ Categoría: Periodismo } & \multicolumn{4}{|c|}{ Asignaturas relacionadas } \\
\hline & & Nivel 1 & Nivel 2 & Nivel 3 & Total \\
\hline Universidad Carlos III & Periodismo & 0 & 1 & & 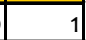 \\
\hline Universidad Católica San Antonio & Periodismo & 0 & 1 & & \\
\hline Universidad de Málaga & Periodismo & 0 & 1 & & \\
\hline Universidad de Sevilla & Periodismo & 0 & 0 & & \\
\hline Universidad del País Vasco & Periodismo & 의 & 1 & & 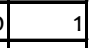 \\
\hline Universidad Francisco de Vitoria & Periodismo & 0 & 2 & & D \\
\hline Universidad Nebrija & Periodismo & 요 & 운 & & 1 \\
\hline Universidad San Jorge & Periodismo & 0 & 0 & & 4 \\
\hline Universidade de Santiago de Compostela & Periodismo & 0 & 1 & & \\
\hline Universitat Internacional de Catalunya & Periodismo & 0 & 1 & & \\
\hline Universitat Jaume I & Periodismo & 0 & 1 & & \\
\hline Universitat Rovira i Virgili & Periodismo & 0 & 0 & & 1 \\
\hline & & 0 & 9 & & 13 \\
\hline
\end{tabular}

Fuente: elaboración propia a partir de datos de las páginas web de las universidades.

Tabla 12. Grados en Publicidad y Relaciones Públicas en cuyos planes de estudio se incluyen asignaturas relacionadas.

\begin{tabular}{|c|c|c|c|c|c|}
\hline \multirow[b]{2}{*}{ Universidad } & \multirow[b]{2}{*}{ Categoría: Publicidad y Relaciones Públicas } & \multicolumn{4}{|c|}{ Asignaturas relacionadas } \\
\hline & & Nivel 1 & Nivel 2 & Nivel 3 & Total \\
\hline Universidad Francisco de Vitoria & Publicidad & 1 & 5 & 4 & 10 \\
\hline ESIC, BUSINESS\&MARKETING SCHOOL (Madrid) & Publicidad y Relaciones Públicas & 1 & 1 & 2 & 4 \\
\hline UDIMA-Universidad a Distancia de Madrid & Publicidad y Relaciones Públicas & 0 & 3 & 1 & 4 \\
\hline Universidad Cardenal Herrera-CEU & Publicidad y Relaciones Públicas & 0 & 1 & 2 & 3 \\
\hline Universidad Católica San Antonio & Publicidad y Relaciones Públicas & 0 & 2 & 1 & 3 \\
\hline Universidad CEU San Pablo & Publicidad y Relaciones Públicas & 0 & 0 & 2 & 2 \\
\hline Universidad Complutense de Madrid & Publicidad y Relaciones Públicas & 0 & 1 & 2 & 3 \\
\hline Universidad de Alicante & Publicidad y Relaciones Públicas & 1 & 2 & 0 & 3 \\
\hline Universidad de Cádiz & Publicidad y Relaciones Públicas & 0 & 1 & 1 & 2 \\
\hline Universidad de Málaga & Publicidad y Relaciones Públicas & 0 & 1 & 2 & 3 \\
\hline Universidad de Murcia & Publicidad y Relaciones Públicas & 0 & 2 & 0 & 2 \\
\hline Universidad de Navarra & Publicidad y Relaciones Públicas & 0 & 4 & 1 & 5 \\
\hline Universidad de Sevilla & Publicidad y Relaciones Públicas & 0 & 3 & 0 & 3 \\
\hline Universidad de Valladolid & Publicidad y Relaciones Públicas & 1 & 1 & 2 & 4 \\
\hline Universidad de Vigo & Publicidad y Relaciones Públicas & 0 & 2 & 1 & 3 \\
\hline Universidad del País Vasco & Publicidad y Relaciones Públicas & 0 & 5 & 2 & 7 \\
\hline Universidad Europea Miguel de Cervantes (UEMC) & Publicidad y Relaciones Públicas & 0 & 3 & 0 & 3 \\
\hline Universidad Nebrija & Publicidad y Relaciones Públicas & 0 & 1 & 0 & 1 \\
\hline Universidad Pontificia Salamanca & Publicidad y Relaciones Públicas & 1 & 2 & 1 & 4 \\
\hline Universidad Rey Juan Carlos & Publicidad y Relaciones Públicas & 1 & 1 & 2 & 4 \\
\hline Universidad San Jorge & Publicidad y Relaciones Públicas & 0 & 1 & 0 & 1 \\
\hline Universitat Abat Oliba CEU & Publicidad y Relaciones Públicas & 0 & 1 & 2 & 3 \\
\hline Universitat de Barcelona & Publicidad y Relaciones Públicas & 0 & 1 & 0 & 1 \\
\hline Universitat de Girona & Publicidad y Relaciones Públicas & 1 & 4 & 4 & 9 \\
\hline Universitat de Vic & Publicidad y Relaciones Públicas & 0 & 4 & 0 & 4 \\
\hline Universitat Internacional de Catalunya & Publicidad y Relaciones Públicas & 0 & 0 & 1 & 1 \\
\hline Universitat Jaume I & Publicidad y Relaciones Públicas & 0 & 4 & 0 & 4 \\
\hline Universitat Pompeu Fabra (UPF) & Publicidad y Relaciones Públicas & 1 & 6 & 3 & 10 \\
\hline Universitat Rovira i Virgili & Publicidad y Relaciones Públicas & 0 & 1 & 1 & 2 \\
\hline CESINE & Publicidad, Relaciones Públicas y Marketing & 0 & 2 & 2 & 4 \\
\hline Universitat Ramon Llull & Publicidad, Relaciones Públicas y Marketing & 0 & 4 & 3 & 7 \\
\hline Universidad Europea Canarias & Comunicación Publicitaria & 1 & 5 & 2 & 8 \\
\hline & & 9 & 74 & 44 & 127 \\
\hline
\end{tabular}

Fuente: elaboración propia a partir de datos de las páginas web de las universidades. 
RLCS, Revista Latina de Comunicación Social, 77, 143-178

[Investigación] DOI: 10.4185/RLCS-2020-1453 | ISSN 1138-5820 | Año 2020

Tabla 13. Grados en Comunicación en cuyos planes de estudio se incluyen asignaturas relacionadas.

\begin{tabular}{|c|c|c|c|c|c|}
\hline \multirow[b]{2}{*}{ Universidad } & \multirow[b]{2}{*}{ Categoría: Comunicación } & \multicolumn{4}{|c|}{ Asignaturas relacionadas } \\
\hline & & Nivel 1 & Nivel 2 & Nivel 3 & Total \\
\hline \begin{tabular}{|l|} 
Universidad Camilo José Cela \\
\end{tabular} & Comunicación & o & & & 2 \\
\hline Universidad Católica San Antonio & Comunicación & 0 & 2 & 2 & 2 \\
\hline \begin{tabular}{|l|} 
Universitat Oberta de Catalunya \\
\end{tabular} & Comunicación & 0 & 2 & 2 & 4 \\
\hline UNIR Universidad Internacional La Rioja & Comunicación & 0 & 2 & 0 & 2 \\
\hline \begin{tabular}{|l|} 
Universidad CEU San Pablo \\
\end{tabular} & Comunicación Digital & 0 & 0 & & 1 \\
\hline Universidad San Jorge & Comunicación Digital & 0 & 1 & t] & 2 \\
\hline ESIC, BUSINESS\&MARKETING SCHOOL (Valencia) & Comunicación y Relaciones Públicas & 0 & 4 & 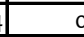 & 4 \\
\hline Universidad Pontificia Salamanca & Marketing y Comunicación & 0 & 0 & D. & 3 \\
\hline Universitat de Vic & Marketing y Comunicación Empresarial & 0 & 2 & & 4 \\
\hline Universitat Ramon Llull & Periodismo y Comunicación Corporativa & 0 & 1 & 0 & 1 \\
\hline & & 의 & 15 & 10 & 25 \\
\hline
\end{tabular}

Fuente: elaboración propia a partir de datos de las páginas web de las universidades.

Tabla 14. Grados de la categoría Otras en cuyos planes de estudio se incluyen asignaturas relacionadas.

\begin{tabular}{|l|l|r|r|r|r|}
\cline { 3 - 6 } & \multicolumn{3}{c}{ Asignaturas relacionadas } \\
\hline Universidad & Categoría: Otras & Nivel 1 & Nivel 2 & Nivel 3 & Total \\
\hline Escuela Universitaria Formatic Barcelona & Dirección en Relaciones Públicas y Marketing & 0 & 1 & 0 & 1 \\
\hline Bau Centro Universitario de Diseño de Barcelona & Diseño & 0 & 1 & 0 & 1 \\
\hline Universidad Autónoma de Madrid & Lenguas Modernas, Cultura y Comunicación & 0 & 0 & 1 & 1 \\
\hline EUNCET Business School - UPC & Marketing y Comunicación Digital & 0 & 1 & 0 & 1 \\
\hline
\end{tabular}

Fuente: elaboración propia a partir de datos de las páginas web de las universidades.

Como muestra la tabla 15, son 32 los Grados en Comunicación que tienen tres o más asignaturas relacionadas con Estrategia Creativa y Conceptualización. Estas 32 titulaciones suman 145 asignaturas relacionadas -el $72 \%$ de la muestra-. Sólo 8 de las 32 titulaciones no son de la categoría publicidad y relaciones públicas - cuatro de la categoría comunicación y otras tantas de la de comunicación audiovisual, marcadas en azul en la siguiente tabla-. 
RLCS, Revista Latina de Comunicación Social, 77, 143-178

[Investigación] DOI: 10.4185/RLCS-2020-1453 | ISSN 1138-5820 | Año 2020

Tabla 15. Grados en cuyos planes de estudios se incluyen un mayor número de asignaturas relacionadas con la estrategia y/o la creatividad.

\begin{tabular}{|c|c|c|c|c|c|c|}
\hline & \multirow[b]{2}{*}{ Universidad } & \multirow[b]{2}{*}{ Grado } & \multicolumn{4}{|c|}{ Asignaturas relacionadas } \\
\hline & & & Nivel 1 & Nivel 2 & Nivel 3 & Total \\
\hline 1 & Universidad Francisco de Vitoria & Publicidad & 1 & 5 & 4 & 10 \\
\hline 2 & Universitat Pompeu Fabra (UPF) & Publicidad y Relaciones Públicas & 1 & 6 & 3 & 10 \\
\hline 3 & Universitat de Girona & Publicidad y Relaciones Públicas & 1 & 4 & 4 & 9 \\
\hline 4 & Universidad Europea Canarias & Comunicación Publicitaria & 1 & 5 & 2 & 8 \\
\hline 5 & Universidad del País Vasco & Publicidad y Relaciones Públicas & 0 & 5 & 2 & 7 \\
\hline 6 & Universitat Ramon Llull & Publicidad, Relaciones Públicas y Marketing & 0 & 4 & 3 & 7 \\
\hline 7 & Universitat Pompeu Fabra (UPF) & Comunicación Audiovisual & 0 & 5 & 0 & 5 \\
\hline 8 & Universidad de Navarra & Publicidad y Relaciones Públicas & 0 & 4 & 1 & 5 \\
\hline 9 & Universitat de Lleida & Comunicación y Periodismo Audiovisuales & 0 & 4 & 0 & 4 \\
\hline 10 & ESIC, BUSINESS\&MARKETING SCHOOL (Madrid) & Publicidad y Relaciones Públicas & 1 & 1 & 2 & 4 \\
\hline 11 & UDIMA-Universidad a Distancia de Madrid & Publicidad y Relaciones Públicas & 0 & 3 & 1 & 4 \\
\hline 12 & Universidad de Valladolid & Publicidad y Relaciones Públicas & 1 & 1 & 2 & 4 \\
\hline 13 & Universidad Pontificia Salamanca & Publicidad y Relaciones Públicas & 1 & 2 & 1 & 4 \\
\hline 14 & Universidad Rey Juan Carlos & Publicidad y Relaciones Públicas & 1 & 1 & 2 & 4 \\
\hline 15 & Universitat de Vic & Publicidad y Relaciones Públicas & 0 & 4 & 0 & 4 \\
\hline 16 & Universitat Jaume I & Publicidad y Relaciones Públicas & 0 & 4 & 0 & 4 \\
\hline 17 & CESINE & Publicidad, Relaciones Públicas y Marketing & 0 & 2 & 2 & 4 \\
\hline 18 & Universitat Oberta de Catalunya & Comunicación & 0 & 2 & 2 & 4 \\
\hline 19 & ESIC, BUSINESS\&MARKETING SCHOOL (Valencia) & Comunicación y Relaciones Públicas & 0 & 4 & o & 4 \\
\hline 20 & Universitat de Vic & Marketing y Comunicación Empresarial & 0 & 2 & 2 & 4 \\
\hline 21 & Universidad del País Vasco & Comunicación Audiovisual & 0 & 3 & 0 & 3 \\
\hline 22 & Universitat Politècnica de València & Comunicación Audiovisual & 0 & 3 & 0 & 3 \\
\hline 23 & Universidad Cardenal Herrera-CEU & Publicidad y Relaciones Públicas & 0 & 1 & 2 & 3 \\
\hline-4 & Universidad Católica San Antonio & Publicidad y Relaciones Públicas & 0 & 2 & 1 & 3 \\
\hline 25 & Universidad Complutense de Madrid & Publicidad y Relaciones Públicas & 0 & 1 & 2 & 3 \\
\hline 26 & Universidad de Alicante & Publicidad y Relaciones Públicas & 1 & 2 & 0 & 3 \\
\hline 27 & Universidad de Málaga & Publicidad y Relaciones Públicas & 0 & 1 & 2 & 3 \\
\hline 8 & Universidad de Sevilla & Publicidad y Relaciones Públicas & 0 & 3 & 0 & 3 \\
\hline 29 & Universidad de Vigo & Publicidad y Relaciones Públicas & 0 & 2 & 1 & 3 \\
\hline 30 & Universidad Europea Miguel de Cervantes (UEMC) & Publicidad y Relaciones Públicas & 0 & 3 & o & 3 \\
\hline 31 & Universitat Abat Oliba CEU & Publicidad y Relaciones Públicas & 0 & 1 & 2 & 3 \\
\hline 32[ & Universidad Pontificia Salamanca & Marketing y Comunicación & 요 & 0 & 3 & 3 \\
\hline
\end{tabular}

Fuente: elaboración propia a partir de datos de las páginas web de las universidades.

En la figura 2 se incluyen las seis universidades cuyos Grados ofertados en Publicidad y Relaciones Públicas incluyen un mayor número de asignaturas relacionadas con Estrategia Creativa y Conceptualización:

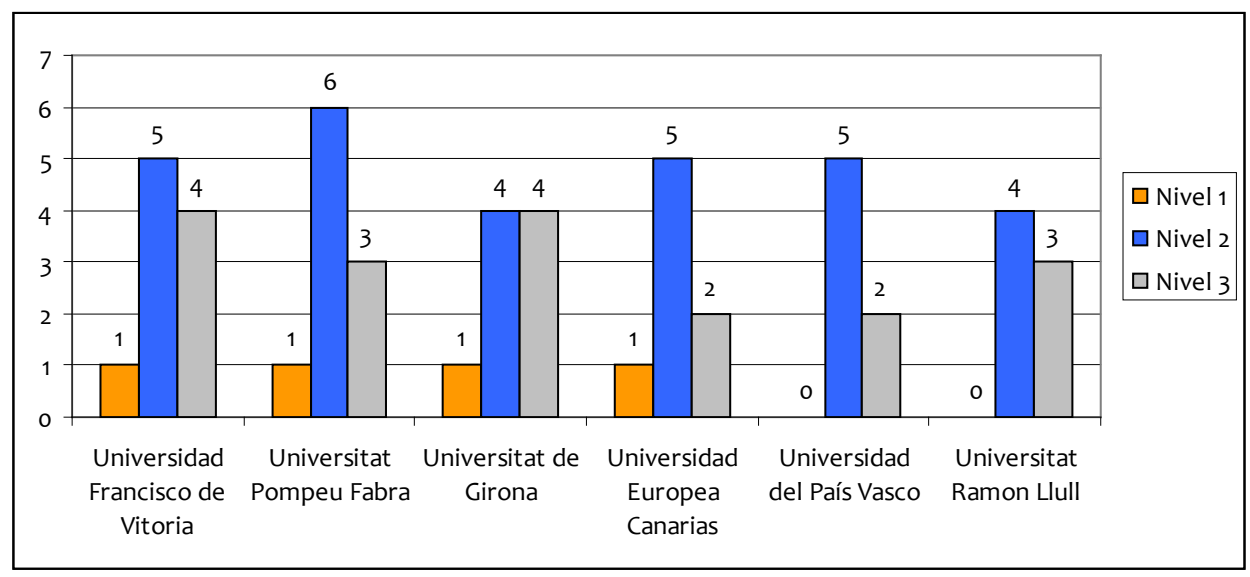

Figura 2: principales universidades con asignaturas relacionadas con la estrategia y/o la creatividad en los planes de estudios de los Grados en Publicidad y Relaciones Públicas.

Fuente: elaboración propia a partir de datos de las páginas web de las universidades. 
Los Grados en Publicidad y Publicidad y Relaciones Públicas de la Universidad Francisco de Vitoria y la Universitat Pompeu Fabra, respectivamente, son los que más asignaturas coincidentes tienen, 10 cada uno de ellos. Les siguen el Grado en Publicidad y Relaciones Públicas de la Universitat de Girona y el Grado en Comunicación Publicitaria de la Universidad Europea Canarias, con 9 y 8 asignaturas, respectivamente.

Pasando a analizar las asignaturas del nivel 1, podemos decir que no existe un consenso en cuanto a las nomenclaturas de las asignaturas; de las 9 encontradas sólo dos comparten nombre -Estrategias Creativas en Publicidad-. Lo que sí coincide es el Grado de procedencia de las mismas, de la categoría publicidad y relaciones públicas. Los nombres de las asignaturas son los siguientes ${ }^{4}$ :

- Estrategia Creativa Publicitaria.

- Estrategia Creativa y Conceptualización.

- Estrategia y Creatividad.

- Estrategias Creativas en Publicidad.

- Estrategias de Creación de Marca y Campaña.

- Estrategias Innovadoras.

- Ideación y Estrategia de Campañas.

- Ideas, Métodos y Estrategias Creativas: el plan o proyecto creativo.

En la tabla 16 se indican los datos de las asignaturas de nivel 1 encontradas:

Tabla 16. Información sobre las asignaturas relacionadas con la estrategia y/o la creatividad de nivel 1 .

\begin{tabular}{|c|c|c|c|c|c|}
\hline Universidad & Grado & Curso & ECTS & Tipo & Asignatura \\
\hline Universidad Pontificia Salamanca & Publicidad y Relaciones Públicas & 3 & 6 & Obligatoria & Estrategia Creativa Publicitaria \\
\hline Universidad de Alicante & Publicidad y Relaciones Públicas & 3 & 6 & Obligatoria & Estrategia Creativa y Conceptualización \\
\hline Universitat Pompeu Fabra & Publicidad y Relaciones Públicas & $3-4$ & 4 & Optativa & Estrategia y Creatividad \\
\hline ESIC (Madrid) & Publicidad y Relaciones Públicas & 2 & 6 & Obligatoria & Estrategias Creativas en Publicidad \\
\hline Universidad Rey Juan Carlos & Publicidad y Relaciones Públicas & 2 & 6 & Obligatoria & Estrategias Creativas en Publicidad \\
\hline Universitat de Girona & Publicidad y Relaciones Públicas & 4 & 6 & Obligatoria & Estrategias de Creación de Marca y de campaña \\
\hline \begin{tabular}{|l|} 
Universidad Europea Canarias \\
\end{tabular} & Comunicación Publicitaria & 3 & 6 & Optativa & Estrategias Innovadoras \\
\hline Universidad Francisco de Vitoria & Publicidad & 3 & 12 & Obligatoria & Ideación y Estrategia de Campañas \\
\hline Universidad de Valladolid & Publicidad y Relaciones Públicas & 3 & 6 & Obligatoria & Ideas, Métodos y Estrategias Creativas: el plan o proyecto creativo \\
\hline
\end{tabular}

Fuente: elaboración propia a partir de datos de las páginas web de las universidades

Como vemos, 7 de las 9 asignaturas son obligatorias y 2 tienen el carácter de optativas, también 7 de las 9 se encuentran en tercer o cuarto curso y otras tantas corresponden a 6 créditos ECTS -en el caso de la Universidad Francisco de Vitoria, la asignatura Ideación y Estrategia de Campañas cuenta con 12 créditos al ser anual y la optativa de la Universidad Pompeu Fabra equivale a 4 créditos ECTS-

Pasando al nivel 2 de asignaturas relacionadas ${ }^{5}$, no existe un consenso en cuanto a la nomenclatura de las asignaturas, por lo que las 131 asignaturas de este nivel representan 90 nombres diferentes. Las nomenclaturas más populares pueden verse en la figura 3.

\footnotetext{
${ }^{4}$ Pueden consultarse las guías docentes de las asignaturas de primer nivel para el curso académico 2016-2017 en el siguiente enlace: http://bit.ly/guias-docentes-nivell

${ }^{5}$ Puede verse el listado de asignaturas de los niveles 2 y 3 en el siguiente enlace: $\underline{\text { http://bit.ly/asignaturas-niveles } 2 \mathrm{y} 3}$.
} 
RLCS, Revista Latina de Comunicación Social, 77, 143-178

[Investigación] DOI: 10.4185/RLCS-2020-1453 | ISSN 1138-5820 | Año 2020

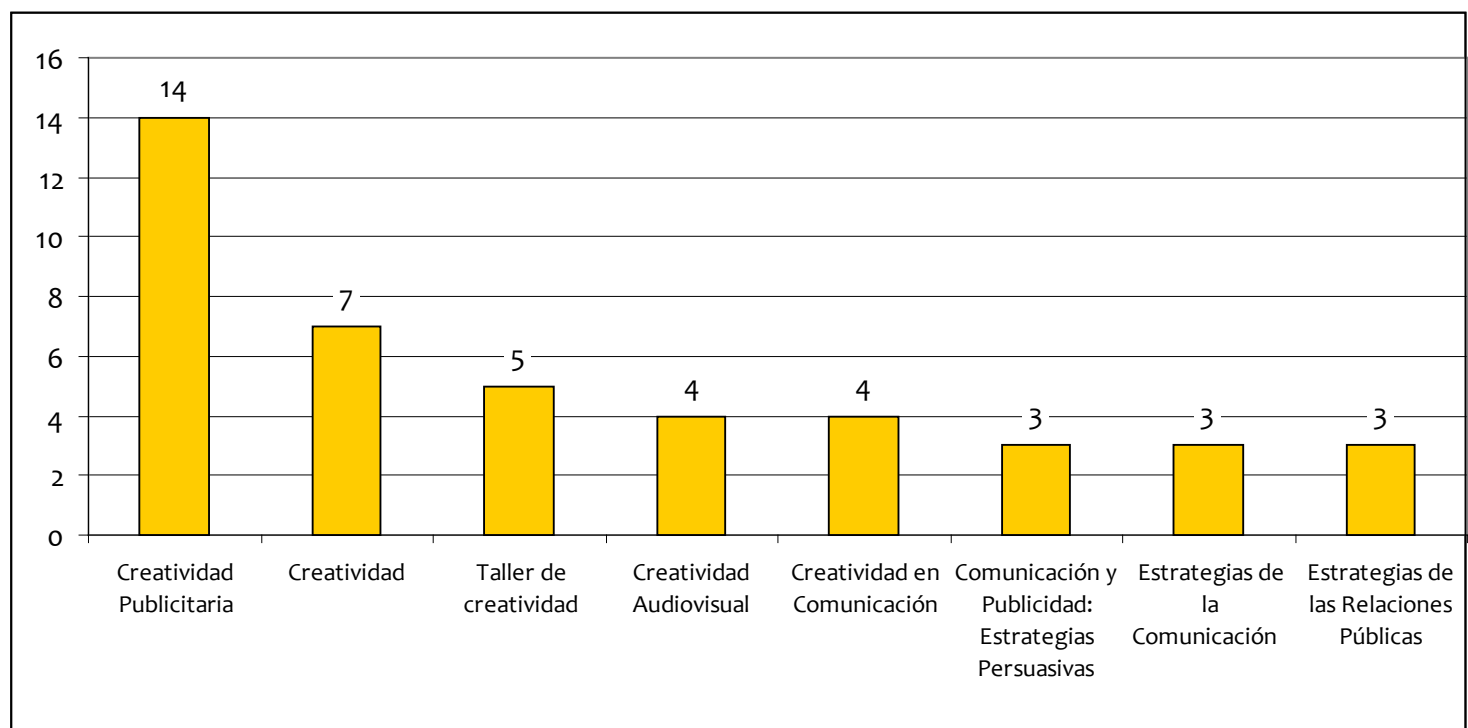

Figura 3: asignaturas del nivel 2 con mayor presencia en los planes de estudio.

Fuente: elaboración propia a partir de datos de las páginas web de las universidades.

La creatividad y la estrategia tienen una presencia similar en los planes de estudio de los Grados en Comunicación, al menos en lo que se refiere a incluir estas palabras en la nomenclatura de la asignatura, si bien existe más consenso a la hora de definir las asignaturas relacionadas con la creatividad, como apunta el gráfico anterior -las cinco asignaturas en cuyo nombre existe más consenso son de creatividad-.

La palabra creatividad aparece en 52 nomenclaturas y la palabra estrategia en 51. La palabra creación aparece en 21 casos, si bien hay que precisar que lo hace desde un enfoque más centrado en técnicas y herramientas: creación multimedia, creación radiofónica, talles de creación, creación y diseño, etc. La palabra ideación aparece en cinco ocasiones, mientras que la innovación publicitaria tiene una asignatura en el Grado en Publicidad y Relaciones Públicas de la Universitat de Girona.

Por último, la palabra conceptualización sólo aparece una vez, en la asignatura Conceptualización en Publicidad y las Relaciones Públicas del Grado en Publicidad, Relaciones Públicas y Marketing de la Universita Ramon Llull.

En cuanto al nivel 3, como se aprecia en la figura 4, Escritura Creativa y Pensamiento Creativo son las asignaturas que más se repiten -en 7 y 5 ocasiones, respectivamente-. No obstante, a pesar de estos dos primeros puestos, parece que en este nivel existe más consenso a la hora de dar nombre a las asignaturas relacionadas con lo estratégico: entre las 11 asignaturas que se repiten dos o más veces, 9 corresponden al ámbito de la estrategia. 


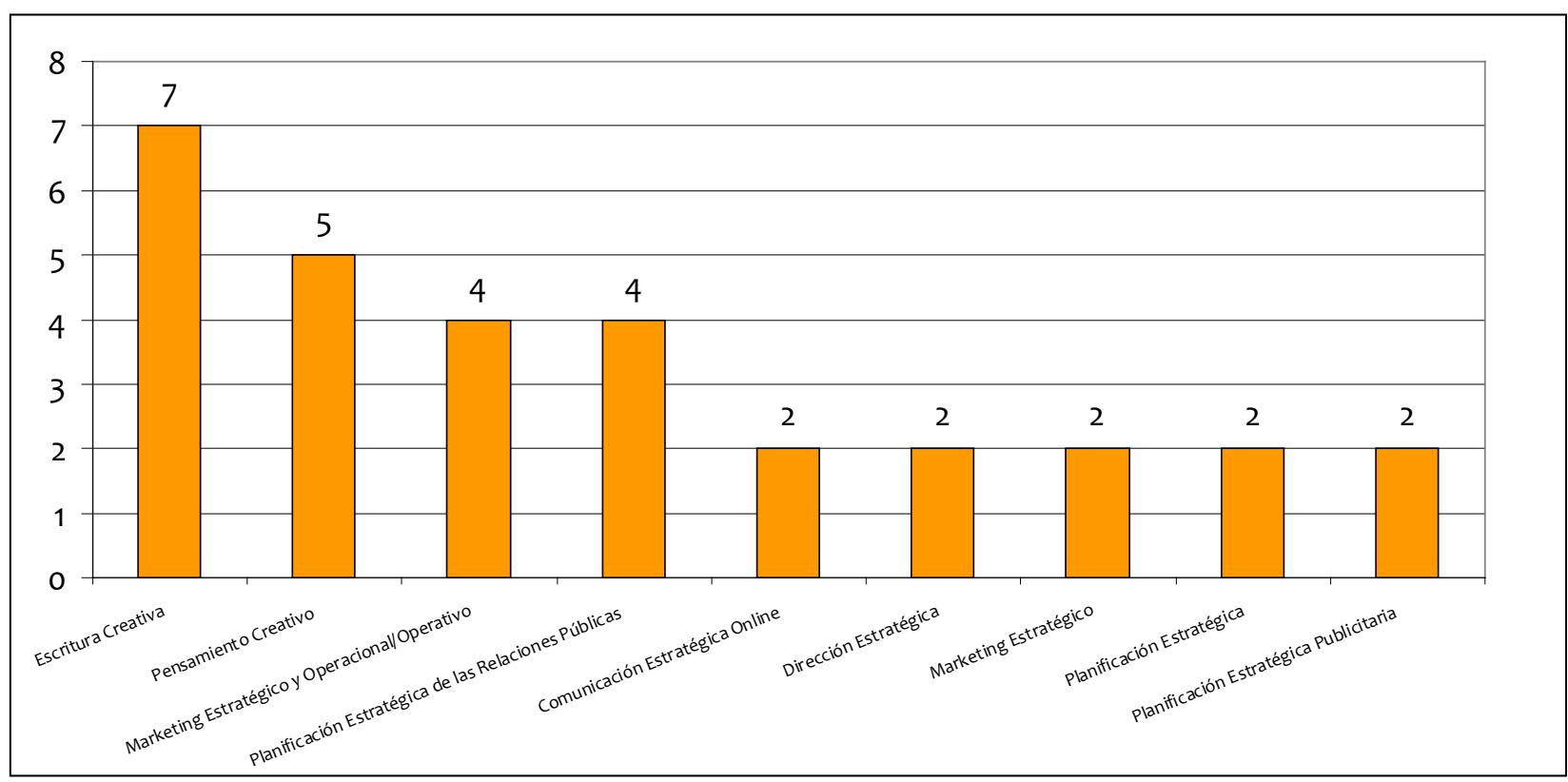

Figura 4: asignaturas del nivel 3 con mayor presencia en los planes de estudio.

Fuente: elaboración propia a partir de datos de las páginas web de las universidades.

El adjetivo creativo/a aparece en 21 ocasiones, mientras que el adjetivo estratégico/a se incluye en 42 nombres de asignaturas. En total, encontramos los dos adjetivos en 63 casos, siendo el total de asignaturas relacionadas en este nivel de 62, puesto que en un caso el nombre de la asignatura incluye los dos adjetivos: Planificación Estratégica y Creativa, del Grado en Comunicación de la Universitat Oberta de Catalunya. Es por este motivo por el que incluimos también esta asignatura en el análisis del plan de estudios de las asignaturas más relacionadas -las del nivel 1- que presentamos a continuación.

Centrándonos en las asignaturas directamente relacionadas con la estrategia creativa y la conceptualización, son 9 las que hemos encontrado, como mostraba la tabla 16. Además de estas asignaturas identificadas en el nivel 1 de relación, incluiremos también en este análisis dos asignaturas del nivel 3, por la estrecha relación que guardan con la estrategia creativa y la conceptualización: Planificación Estratégica de la Publicidad y de las Relaciones Públicas: el planner y Planificación Estratégica y Creativa.

Estas 11 asignaturas proceden, en su mayoría, de Grados en Publicidad y Relaciones Públicas -sólo la asignatura Planificación Estratégica y Creativa procede del Grado en Comunicación. Si la muestra total de Grados en Publicidad y Relaciones Públicas era de 32 titulaciones, sólo en 9 casos encontramos asignaturas directamente relacionadas con la estrategia creativa y la conceptualización, es decir en el 31,3\% de la muestra. En seis casos las asignaturas se imparten en instituciones privadas y en cinco casos en universidades públicas.

Con respecto a las guías docentes para el curso académico 2016-2017 de estas asignaturas, se analizan los que están disponibles en las páginas web de las universidades. En la tabla 17 se incluyen los enlaces a las guías docentes de las asignaturas de las 9 titulaciones que las tienen online -en los casos de la asignatura optativa en la Universitat Pompeu Fabra y la asignatura obligatoria en ESIC Madrid, en la página web de la titulación no se incluye información detallada de las asignaturas-. 
RLCS, Revista Latina de Comunicación Social, 77, 143-178

[Investigación] DOI: 10.4185/RLCS-2020-1453 | ISSN 1138-5820 | Año 2020

Tabla 17. Enlaces a las guías docentes de las asignaturas relacionadas de nivel 1.

\begin{tabular}{|c|c|c|c|c|c|c|c|}
\hline Universidad & Tipo & Grado & Curso & ECTS & Tipo & Asignatura & Enlace \\
\hline $\begin{array}{l}\text { Universidad Pontificia } \\
\text { Salamanca }\end{array}$ & Privada & $\begin{array}{l}\text { Publicidad y } \\
\text { Relaciones Públicas }\end{array}$ & 3 & 6 & Obligatoria & $\begin{array}{l}\text { Estrategia Creativa } \\
\text { Publicitaria }\end{array}$ & http://bit.ly/upsa-ecc \\
\hline $\begin{array}{l}\text { Universidad de } \\
\text { Alicante }\end{array}$ & Pública & \begin{tabular}{|l} 
Publicidad y \\
Relaciones Públicas
\end{tabular} & 3 & 6 & Obligatoria & \begin{tabular}{|l} 
Estrategia Creativa y \\
Conceptualización
\end{tabular} & http://bit.ly/ua-ecc \\
\hline $\begin{array}{l}\text { Universitat Pompeu } \\
\text { Fabra }\end{array}$ & Pública & $\begin{array}{l}\text { Publicidad y } \\
\text { Relaciones Públicas }\end{array}$ & $3-4$ & 4 & Optativa & Estrategia y Creatividad & - \\
\hline ESIC (Madrid) & Privada & \begin{tabular}{|l} 
Publicidad y \\
Relaciones Públicas
\end{tabular} & 2 & 6 & Obligatoria & $\begin{array}{l}\text { Estrategias Creativas en } \\
\text { Publicidad }\end{array}$ & - \\
\hline $\begin{array}{l}\text { Universidad Rey Juan } \\
\text { Carlos }\end{array}$ & Pública & $\begin{array}{l}\text { Publicidad y } \\
\text { Relaciones Públicas }\end{array}$ & 2 & 6 & Obligatoria & $\begin{array}{l}\text { Estrategias Creativas en } \\
\text { Publicidad }\end{array}$ & Se descarga desde http://bit.ly/urjc-ecc \\
\hline Universitat de Girona & Pública & \begin{tabular}{|l} 
Publicidad y \\
Relaciones Públicas
\end{tabular} & 4 & 6 & Obligatoria & $\begin{array}{l}\text { Estrategias de Creación de } \\
\text { Marca y de campaña }\end{array}$ & http://bit.ly/udg-ecc \\
\hline $\begin{array}{l}\text { Universidad Europea } \\
\text { Canarias }\end{array}$ & Privada & $\begin{array}{l}\text { Comunicación } \\
\text { Publicitaria }\end{array}$ & 3 & 6 & Optativa & Estrategias Innovadoras & http://bit.ly/ue-ecc \\
\hline $\begin{array}{l}\text { Universidad Francisco } \\
\text { de Vitoria }\end{array}$ & Privada & Publicidad & 3 & 12 & Obligatoria & $\begin{array}{l}\text { Ideación y Estrategia de } \\
\text { Campañas }\end{array}$ & http://bit.ly/ufv-ecc \\
\hline $\begin{array}{l}\text { Universidad de } \\
\text { Valladolid }\end{array}$ & Pública & $\begin{array}{l}\text { Publicidad y } \\
\text { Relaciones Públicas }\end{array}$ & 3 & 6 & Obligatoria & $\begin{array}{l}\text { Ideas, Métodos y Estrategias } \\
\text { Creativas: el plan o proyecto } \\
\text { creativo }\end{array}$ & http://bit.ly/uva-ecc \\
\hline $\begin{array}{l}\text { Universitat Ramon } \\
\text { Llull }\end{array}$ & Privada & $\begin{array}{l}\text { Publicidad y } \\
\text { Relaciones Públicas }\end{array}$ & 3 & 3 & Obligatoria & $\begin{array}{l}\text { Planificación Estratégica de la } \\
\text { Publicidad y las Relaciones } \\
\text { Públicas: el planner }\end{array}$ & http://bit.ly/blanquerna-ecc \\
\hline $\begin{array}{l}\text { Universitat Oberta de } \\
\text { Catalunya }\end{array}$ & Privada & Comunicación & $3-4$ & 3 & Optativa & $\begin{array}{l}\text { Planificación Estratégica y } \\
\text { Creativa }\end{array}$ & http://bit.ly/uoc-ecc \\
\hline
\end{tabular}

Fuente: elaboración propia a partir de datos de las páginas web de las universidades.

\section{Discusión}

La estrategia y la creatividad son materias fundamentales en el Grado en Publicidad y Relaciones Públicas, tal y como se ha podido comprobar en el análisis de competencias, destrezas, habilidades y perfiles profesionales indicados por ANECA (2005) así como en los resultados del estudio que hemos llevado a cabo sobre las asignaturas en los Grados en Comunicación: todos los Grados de la categoría publicidad y relaciones públicas tienen asignaturas relacionadas en los distintos niveles.

Sin embargo, si bien todas las asignaturas similares a Estrategia Creativa y Conceptualización identificadas pertenecen a estudios de Publicidad y Relaciones Públicas, éstas resultan escasas, ya que sólo el $28 \%$ de los Grados en la categoría publicidad y relaciones públicas tienen asignaturas relacionadas con la estrategia creativa y la conceptualización.

Por su parte, ni la estrategia ni la creatividad ni la combinación de ambas tienen presencia destacada en los planes de estudios de los Grados en Comunicación Audiovisual y Periodismo: el 63\% de las asignaturas identificadas se encuentran en titulaciones de la categoría publicidad y relaciones públicas. Por tanto, podemos decir que se cumple la hipótesis principal en tanto en cuanto las asignaturas existentes en los Grados en Comunicación que combinan el estudio de la creatividad y la estrategia son escasas.

Otro aspecto que consideramos conveniente recalcar es la necesidad de una revisión de las nomenclaturas de las asignaturas de creatividad, siguiendo las propuestas de Stuhlafaut y Berman (2009) y de Alegre y Roca (2012), corroboradas con el estudio que hemos llevado a cabo sobre las asignaturas de creatividad y estrategia publicitarias clasificadas en varios niveles. Este trabajo de coordinación facilitará la movilidad de los estudiantes en el ámbito nacional y contribuirá a la delimitación de los contenidos de cada asignatura, puesto que en ocasiones éstos se solapan, especialmente cuando las asignaturas forman parte de la misma materia. 
Excepto la asignatura Estrategias de Creación de Marca y de Campañas, del Grado en Publicidad y Relaciones Públicas en la Universitat de Girona, que se ocupa en parte de la creación y gestión de la marca, el resto de asignaturas cuyos planes de estudio se han revisado se centran en el proceso creativo desde la definición de los objetivos publicitarios hasta la evaluación de los resultados de las acciones publicitarias diseñadas, enfocándose principalmente en dos figuras profesionales: el creativo y el planner.

Además, los contenidos de estas asignaturas demuestran que la creatividad publicitaria, hoy en día, no se puede limitar a la actividad de copys y directores de arte en los departamentos creativos de las agencias, sino que se entiende como una cualidad general de cualquier profesional de la comunicación publicitaria, por lo que desde la Universidad se procura dar esta visión holística integrando el enfoque creativo en los sistemas y procesos publicitarios y destacando la aplicabilidad de la creatividad en todas y cada una de sus fases.

Con respecto a las competencias, destacamos las siguientes genéricas y específicas, por ser las más populares en las guías docentes de las asignaturas de estrategia creativa y conceptualización:

1. Generales: trabajo en equipo.

2. Generales: capacidad de expresión oral y escrita, de manera correcta, rica y fluida.

3. Generales: manejo de las TIC como herramientas básicas y habituales de trabajo y aprovechar sus potencialidades en el ámbito profesional.

4. Generales: capacidad para la ideación, la creatividad y la innovación.

1. Específicas: actitud estratégica y capacidad de conceptualización.

2. Específicas: planificación y diseño de estrategias publicitarias a partir de unos objetivos.

3. Específicas: conocimiento de las funciones de las figuras profesionales (creativo y planner) y de las últimas tendencias en creatividad publicitaria.

4. Específicas: investigación y comprensión del consumidor.

En el curso 2016-2017 todas las asignaturas revisadas se basan en metodologías participativas que involucran al alumnado, con un peso importante de las actividades prácticas en un proceso de evaluación continua. No obstante, la clase magistral basada en una presentación interactiva sigue siendo la práctica más común a la hora de abordar los contenidos teóricos, por lo que podemos decir que, en estos casos, la conversión hacia un modelo integral del aprendizaje cooperativo se encuentra en una fase intermedia.

\section{Conclusiones}

Si la creación del EEES fue motivada por la necesidad de un mayor contacto con la realidad profesional por parte de la Academia, esta búsqueda de sinergias con la industria debe ser la que guíe cualquier metodología docente $\mathrm{y}$, desde nuestro punto de vista, desde la Universidad deben crearse más puentes que permitan y animen a los profesionales a acercarse a los alumnos -y viceversa-, porque los alumnos están deseosos de interactuar con la realidad profesional. En este sentido, como destaca Muela (2012), la materia de creatividad publicitaria:

aunque debe desarrollar una parte teórica sobre los conceptos básicos de esta actividad como

parte fundamental de un proceso encaminado a la resolución de problemas para obtener productos originales, también debe eliminar los mitos asociados a la figura del creativo como genio o iluminado. Ésta es, por tanto, una asignatura enfocada a la profesión, pero también un 
requisito indispensable en cualquier etapa del proceso estratégico de una campaña que, especialmente, se adscribe a un departamento y a unos profesionales que trabajan en grupos creativos. (p. 34)

Como señala el Libro Blanco de las titulaciones de comunicación (ANECA, 2005, p. 337), el profesor de creatividad publicitaria debe provocar en el alumno un "acercamiento a la realidad profesional y al quehacer comunicativo, desde la reflexión y el estudio entendido como el conjunto de sus diferentes acciones, especialmente publicitarias".

El planificador estratégico de la comunicación ha ido ocupando un lugar clave en las estructuras de las agencias e incluso cada vez es más habitual que se incorpore en los departamentos de marketing y comunicación del anunciante (Castelló-Martínez, 2019; Sebastián-Morillas, Martín-Soladana y Clemente-Mediavilla, 2020). Como afirma Gem Romero -Head of Strategy en LOLA MullenLowe Barcelona-, "el pensamiento estratégico es necesario en la publicidad igual que lo es la creatividad [...]. Estrategas o creativos deberíamos ser todos en las distintas fases del proceso publicitario" (Álvarez-Ruíz, 2012, p. 13). La planificación estratégica está consolidándose en España con un elevado nivel de profesionalización, de manera que los planes de estudios de Comunicación deben dar respuesta urgente a estas nuevas necesidades del mercado, poniendo el foco en la figura del planner.

Desde el periodo de recesión económica iniciado en 2008, el anunciante ha reducido su inversión publicitaria -los 5.951,8 millones de euros invertidos en publicidad en medios controlados en 2019 están más cerca de los 5.110 millones de euros de 1999 que de los 7.986 millones de euros de 2007 (InfoAdex, 2020)-, pero invierte de manera más inteligente, demandando soluciones comunicativas integradas que aseguren la medición de la eficacia y rentabilidad, permitan la personalización del mensaje, incorporen la participación del público y su interacción con él y en las que el consumidor sea el protagonista.

La importancia que adquiere el contexto de comunicación para aquellas marcas que ansían alcanzar el estadio de marcas útiles, dando una respuesta franca y duradera en la comunicación persuasiva a las tensiones y preocupaciones sociales del entorno, pone en valor la figura del planner, cuya principal tarea es la de conocer y comprender al consumidor del público destinatario hasta el punto de empatizar con él. El planificador estratégico es el responsable de que las ejecuciones creativas tengan una base sólida gracias a la elección de un eje de comunicación diferencial y a la identificación de un concepto creativo que lo transforme en un mensaje empático, estimulante, sorpresivo y memorable para el público objetivo.

El eje de comunicación es el pilar sobre el que se sustenta cualquier estrategia de comunicación persuasiva integrada (Zomeño, 2014, pp. 211-212; Castelló-Martínez y Del Pino-Romero, 2019). Para conceptualizarlo creativamente, a partir del conocimiento en profundidad del público, se puede recurrir a insights del consumidor, poniéndolos al servicio del mensaje de la marca. A partir de la búsqueda documental sobre la marca y el producto, la competencia, el mercado y el público objetivo, el planificador estratégico es el responsable de estas dos primeras fases del proceso creativo en cualquier estrategia publicitaria. Como afirman Hernández y Pinar (2012):

la creatividad requiere de la planificación estratégica para asegurar la efectividad de sus

mensajes, pues es en la fase estratégica donde se decide qué decir respecto a las marcas y productos, a quién hacerlo y a través de qué medios. (p. 379) 
Este nuevo paradigma de la comunicación persuasiva integrada justifica la necesidad de que la estrategia sea creativa y la creatividad sea estratégica, la importancia de la figura del planner y el apremio a la hora de formar a profesionales de la comunicación con este perfil, adaptando los planes de estudios a la cambiante realidad.

\section{Referencias bibliográficas}

Alegre Rodríguez, I. y Roca Correa, D. (2012). La Enseñanza de la Creatividad en los Estudios de Publicidad de las Universidades Españolas. http://bit.ly/alegre-roca-2012

Álvarez-Ruiz, A. (2012). La magia del planner. Cómo la planificación estratégica puede potenciar la creatividad persuasiva. Esic.

ANECA (2005). Libro Blanco. Títulos de Grado en Comunicación. http://bit.ly/libro-blanco-aneca

Castelló-Martínez, A. (2019). Estado de la planificación estratégica y la figura del planner en España. Los insights como concepto creativo. Revista Mediterránea de Comunicación, 10(2), 2943. http://bit.ly/rmc-castello-planificación

Castelló-Martínez, A. y Del Pino-Romero, C. (2019). De la publicidad a la comunicación persuasiva integrada. Estrategia y empatía. Esic.

Davara, F. J. (1994). Estrategias de comunicación en marketing. Dossat.

De la Torre, S. y Violant, V. (2003). Estrategias creativas en la enseñanza universitaria. Una investigación con metodología de desarrollo. Creatividad y Sociedad, (3), 1-23. http://bit.ly/delatorre-violant-2003

Dineen, Ru, \& Collins, E. (2004). Out of the Box: the Promotion of Creativity in Learners. DATA International Research Conference 2004 Creativity and Innovation.

Estanyol i Casals, E. (2012). La enseñanza de la creatividad aplicada en relaciones públicas, en el mercado de los grados adaptados al EEES. Comunicación y riesgo: Congreso Internacional Asociación Española de Investigación de la Comunicación. http://bit.ly/estanyol-2012

Fernández March, A. (2005). Nuevas metodologías docentes. VV. AA. Materiales para talleres de formación del profesorado de la UPM para el EEES. ANECA-UPM.

Freire, P. (1995). Pedagogía del oprimido. Siglo XXI.

García-Uceda, M. (2001). Las claves de la publicidad. Esic.

González, J., \& Wagenaar, R. (Eds.) (2003). Tuning Educational Structures in Europe. Informe Final. Proyecto Piloto - Fase 1. Universidad de Deusto. http://bit.ly/gonzalez-wagenaar-2003.

Hernández Martínez, C. y Pinar Selva, M. L. (2012). Las bases estratégicas de la creatividad publicitaria. En Cuesta, U. (Coord.). Planificación estratégica y creatividad (pp. 375-391). Esic.

InfoAdex (2020). Estudio InfoAdex de la Inversión Publicitaria en España 2020. $\underline{\text { http://bit.ly/infoadex-2020 }}$ 
Kapferer, J.-N. (1997). Strategic Brand Management. Kogan Page.

Kolg, D. A. (1984). Experiential Learning. Experience as the source of learning and development. Prentice-Hall.

Marín Ibáñez, R. (1989). La formación para la creatividad. Universidad Nacional de Educación a Distancia.

Marta-Lazo, C. (2012). El aprendizaje basado en competencias en la educación universitaria del siglo XXI. En Marta Lazo, C., Agustín Lacruz, M. C. y Ubieto Artur, M. I. Competencias interdisciplinares para la comunicación y la información en la sociedad digital. Icono14, 13-30.

Marta-Lazo, C. (2009). El proceso de reconversión de la comunicación en el EEES. Icono14. Revista de comunicación y tecnologías emergentes, 7(3), 1-7. http://bit.ly/marta-2009 http://dx.doi.org/10.7195/ri14.v7i3.492

Muela Molina, C. (2012). Aprendizaje cooperativo de la creatividad publicitaria a través de anuncios audio y/o visuales. @tic. Revista d'innovació educativa, (8), 33-39. http://bit.ly/muela-2012

Pérez Martínez, V. M., Vadillo Bengoa, N. y McMahon, J. (2011). Experiencias colaborativas en la formación de estudiantes de periodismo: Press Writing and Communication I y Documentación Informativa. En Marta Lazo (Coord.). Retos del profesional de la Comunicación en la Sociedad del Conocimiento. Cuadernos Artesanos de Latina, $\mathrm{n}^{\mathrm{o}}$ 7, (pp. 66-88). Sociedad Latina de Comunicación Social.

Pérez-González, R. A. (1989). Estrategia publicitaria y de las relaciones públicas. Universidad Complutense de Madrid.

Ross, B. \& Richards, J. (2008). A Century of Advertising Education. American Academy of Advertising.

Sebastián-Morillas, A., Martín-Soladana, I., y Clemente-Mediavilla, J. (2020). Importancia de los 'insights' en el proceso estratégico y creativo de las campañas publicitarias. Estudios Sobre El Mensaje Periodístico, 26(1), 339-348. http://bit.ly/sebastian-esmp-2020 https://doi.org/10.5209/esmp.66570

Sierra-Sánchez, J. y Cabezuelo Lorenzo, F. (2010). Recursos de las facultades de comunicación para la formación en competencias en el marco del Espacio Europeo de Educación Superior. Doxa Comunicación, (11), 31-54. http://bit.ly/sierra-cabezuelo-2010

Sivera-Bello, S. (2016). Genios y botellas: cómo se imparte Pensamiento Creativo en la UOC. Opción: Revista de Ciencias Humanas y Sociales, (12), 701-723. http://bit.ly/sivera-bello-2016

Stuhlfaut, Mark W. y Berman, M. (2009). Pedagogic Challenges: The Teaching of Creative Strategy in Advertising. Journal of Advertising Education, 13(2).

Torres Romay, E. (2010). Enseñanza universitaria de la comunicación estratégica en España. Análisis de la repercusión del proceso de adaptación al Espacio Europeo de Educación Superior en la enseñanza de estrategias. Signo y Pensamiento, 29(56), 304-327. http://bit.ly/torres-2010 
Tur-Viñes, V. (2008). La creatividad publicitaria en el Espacio Europeo de Educación Superior. III Simposium de profesores universitarios de creatividad publicitaria, (pp.153-160). http://bit.ly/turvines-2008

Zomeño, D. (2014). Se busca director creativo. adComunica. Revista Científica de Estrategias,Tendencias e Innovación en Comunicación, (8), 225-226. http://bit.ly/zomeno-2014 http://dx.doi.org/10.6035/2174-0992.2014.8.17

\section{Referencias relacionadas}

Aguaded, J. I. (2011). Media education: An international unstoppable phenomenon. The Work of the UN, Europe and Spain in the Field of Educommunication. Comunicar, 19(37), 7-8. http://bit.ly/aguaded-2011 http://dx.doi.org/10.3916/C37-2011-01-01

Ajjan, H. y Hartshorne, R. (2008). Investigating faculty decisions to adopt Web 2.0 technologies: theory and empirical tests. The Internet and Higher Education, 11(2), 71-80. http://bit.ly/ajjanhartshorne-2008 https://doi.org/10.1016/j.iheduc.2008.05.002

Alonso, C. M. (2004). La creatividad publicitaria en la universidad: Reflexiones para la profundización en el ámbito de la docencia. Creatividad y Sociedad, (6), 47-60.

Armendáriz, E. (2015). El nuevo perfil del profesional de la Comunicación y las Relaciones Públicas. Una visión desde la perspectiva del mercado. Revista Internacional de Relaciones Públicas, 5(9), 153-178. http://bit.ly/armendariz-2015 http://dx.doi.org/10.5783/RIRP-9-2015$\underline{09-153-178}$

Arroyo Almaraz, I. (2005). Los valores sociales de la creatividad publicitaria. Icono14. Revista de comunicación y tecnologías emergentes, 3(2), 1-22. $\quad$ http://bit.ly/arroyo-2005 http://dx.doi.org/10.7195/ri14.v3i2.416

Baños González, M. (2005). 'Vayamos por partes...' dijo Jack El Destripador. Icono14. Revista de comunicación y tecnologías emergentes, 3(1), 1-19. http://bit.ly/baños-2005

Benavides Delgado, J. (2012). La investigación en comunicación y publicidad. Questiones Publicitarias, 1(17), 71-93. http://bit.ly/bibliografia-benavides

Camusso, M. Á. (2007). Los mitos de la creatividad en la enseñanza de la creatividad publicitaria: dificultades, paradojas y desafíos. Creatividad y Sociedad, (11), 146-162. http://bit.ly/camusso-2007

Corredor, P. y Farfán, J. (2010). Demandas y formación: Nuevos perfiles profesionales para la Publicidad en España. Pensar la Publicidad, 4(1). 97-116. http://bit.ly/corredor-farfan-2010

Correyero Ruiz, B. y Balandrón Pazos, A. J. (2011). Demandas formativas de los perfiles profesionales emergentes en comunicación en el EEES. En Marta Lazo, C. (Coord.). Retos del profesional de la Comunicación en la Sociedad del Conocimiento. Cuadernos Artesanos de Latina, $n^{\circ}$ 7, (pp. 47-65). Sociedad Latina de Comunicación Social.

Cortés Montalvo, J. A. (2012). La competencia comunicativa en la formación universitaria. En Marta Lazo, C., Agustín Lacruz, M. C. y Ubieto Artur, M. I. Competencias interdisciplinares para la comunicación y la información en la sociedad digital. Icono14, 47-72. 
Earle, L. (2005). Creative Message Strategy as a Framework for Course Planning, Preparation, and Pedagogy Or: Everything I Know About Teaching I Learned from Advertising. Journal of Advertising Education, 9(2).

Fernández Cavia, J. y Sánchez Blanco, C. (2012). Retos de la profesión publicitaria: aportaciones desde la planificación estratégica. Zer. Revista De Estudios De Comunicación, 17(32), 51-71. http://bit.ly/fernandez-sanchez-2012

García García, F. (2006). De la convergencia tecnológica a la convergencia comunicativa en la educación y el progreso. Icono14. Revista de comunicación y tecnologías emergentes, 4(1), 1-19. http://bit.ly/garcia-2006 http://dx.doi.org/10.7195/ri14.v4i1.394

Hellín Ortuño, P. A. (2007). Adaptación al EEES en la Licenciatura de Publicidad y Relaciones Públicas. Una experiencia de innovación docente. Estudos em Comunicaçao, (1), 362-376. http://bit.ly/hellin-2007

Lau, K. \& Ng, Frankie \& Lee, P. (2009). Rethinking the creativity training in design education: a study of creative thinking tools for facilitating creativity development of design students. Art, Design \& Communication in Higher Education, 8(1), 71-81. http://dx.doi.org/10.1386/adch.8.1.71 1

Martín, E. (2014). Tecnocreatividad. adComunica. Revista Científica de Estrategias, Tendencias e Innovación en Comunicación, (8), 213-214. http://bit.ly/martin-eugenio-2014 http://dx.doi.org/10.6035/2174-0992.2014.8.13

Ortiz Sobrino, M. Á. (2009). La reconversación de la formación en comunicación desde el Espacio Europeo de Educación Superior. Icono14. Revista de comunicación y tecnologías emergentes, 7(3), 35-49. http://bit.ly/ortiz-2009 https://doi.org/10.7195/ri14.v7i3.301

Rodrigo Martín, L., García García, F. y Rodrigo Martín, I. (2014). Creatividad y nuevas tecnologías: Las claves de la cultura emprendedora. El papel de la universidad en la formación de intelectuales con espíritu emprendedor. adComunica. Revista Científica de Estrategias, Tendencias e Innovación en Comunicación, (8), 23-37. http://bit.ly/rodrigo-garcia-rodrigo-2014 http://dx.doi.org/10.6035/2174-0992.2014.8.3

Ubieto Artur, M. I., Agustín Lacruz, M. C. y Marta Lazo, C. (2011). El valor de los perfiles de ingreso en la formación de los futuros profesionales de la Información y la Comunicación. En Marta Lazo (Coord.). Retos del profesional de la Comunicación en la Sociedad del Conocimiento. Cuadernos Artesanos de Latina, nº 7, (pp. 89-113). Sociedad Latina de Comunicación Social.

\section{AUTORA:}

\section{Araceli Castelló-Martínez}

Doctora en Comunicación por la Universidad Cardenal Herrera-CEU, Máster en Comunicación Integral por la Universidad Complutense de Madrid, Máster Ejecutivo en Community Management por la Universidad de Alicante y Licenciada en Publicidad y RR.PP. por la Universidad de Alicante, con premio extraordinario de Licenciatura y segundo premio nacional. Profesora Titular de Universidad de la Universidad de Alicante con un sexenio (2009-2014), imparte docencia en los estudios de Publicidad y RR.PP. Ha sido también profesora en la Licenciatura en Publicidad y 
RR.PP. de la Universidad CEU-Cardenal Herrera y ha colaborado como profesora en diversos postgrados. Ha trabajado en agencias de medios (Netthink Carat, Initiative) y soportes digitales (Ya.com, Vocento) en España y Bélgica y realizado numerosas publicaciones sobre planificación publicitaria online, marketing en medios sociales y comunicación digital, como los libros Estrategias empresariales en la Web 2.0. Las redes sociales online y La comunicación en cambio constante o el informe Tendencias en planificación publicitaria online publicado por AIMC. En febrero de 2018, acumula 535 citas, con un índice $\mathrm{H}$ de 12 y un índice 110 de 17.

araceli.castello@ua.es

Orcid ID: http://bit.ly/orcid-araceli-castello

Google Scholar: $\underline{\text { http://bit.ly/scholar-araceli-castello }}$

Web personal: http://www.aracelicastello.com 